\title{
Boosted quantum black hole and black string in M-theory, and quantum correction to Gregory-Laflamme instability
}

\author{
Yoshifumi Hyakutake \\ Faculty of Science, Ibaraki University, \\ Bunkyo 2-1-1, Mito, Ibaraki, 310-8512 Japan \\ E-mail: Yoshifumi.hyakutake.phys@vc.ibaraki.ac.jp
}

ABSTRACT: We take into account higher derivative $R^{4}$ corrections in M-theory and construct quantum black hole and black string solutions in 11 dimensions up to the next leading order. The quantum black string is stretching along the 11th direction and the Gregory-Laflamme instability is examined at the quantum level. Thermodynamics of the boosted quantum black hole and black string are also discussed. Especially we take the near horizon limit of the quantum black string and investigate its instability quantitatively.

KEYwords: Black Holes in String Theory, Gauge-gravity correspondence, Black Holes

ARXIV EPRINT: 1503.05083 


\section{Contents}

1 Introduction 1

2 Quantum black hole and black string in M-theory 2

2.1 Brief review of higher derivative corrections in M-theory 2

2.2 Quantum black hole 3

2.3 Quantum black string 6

2.4 Gregory-Laflamme instability of the quantum black string 8

3 Boosted quantum black hole and black string in M-theory 9

$\begin{array}{ll}3.1 & \text { Boosted quantum black hole }\end{array}$

$\begin{array}{lll}3.2 & \text { Boosted quantum black string } & 10\end{array}$

3.3 Gregory-Laflamme instability of the boosted quantum black string 13

4 Near horizon limit of the boosted quantum black string and its instability 14

$\begin{array}{lll}5 & \text { Conclusion and discussion } & 17\end{array}$

A Calculations on quantum black hole $\quad 19$

$\begin{array}{lll}\text { A.1 Explicit values of tensors } & 19\end{array}$

$\begin{array}{lll}\text { A.2 Plots of } A_{1}(x) & 20\end{array}$

B Calculations on quantum black string $\quad 20$

B.1 Explicit values of tensors 20

B.2 Solution of eq. (2.19) 22

B.3 Plots of $F_{1}(x) \quad 23$

\section{Introduction}

One of challenging problems in theoretical physics is to reveal the nature of the quantum gravity. Especially the superstring theory is a good candidate for the quantum gravity in which properties of black holes have been studied enormously. In this paper we will study the quantum aspects of the black hole and the black string in superstring theory and M-theory.

The type IIA superstring theory is perturbatively defined in 10 dimensions, and its low energy limit is described by the type IIA supergravity. This theory contains black hole solution which possesses $\mathrm{SO}(9)$ rotation symmetry. Interestingly the strong coupling limit of the type IIA superstring theory is believed to be described by 11 dimensional Mtheory $[1,2]$. The 11 th direction is compactified on a circle and its radius is proportional to the string coupling constant. The low energy limit of the M-theory is approximated by the 11 dimensional supergravity [3], and it contains black hole solution which has $\mathrm{SO}(10)$ rotation symmetry. Since the type IIA supergravity is obtained by Kaluza-Klein reduction 
of the 11 dimensional supergravity [4], the 10 dimensional black hole with $\mathrm{SO}(9)$ symmetry is identified with a 11 dimensional black string which is stretching along the 11th direction.

The black string is stable against perturbation when the radius of the compactified circle is small. However, as we enlarge the circle, it becomes unstable at the critical radius and starts to transit to the black hole [5]. This phenomenon is called Gregory-Laflamme instability and has been studied since 1993 [6]. In 2000, the fate of this instability is analyzed and the existence of non-uniform black string is discussed in ref. [7]. After this work, there appeared several analytic studies and numerical simulations in order to resolve the final state of the unstable black string [8]-[14]. These show that, beyond the critical radius, the black string transit into the non-uniform string and finally pinch off. It is also revealed that the fate of the unstable black string depends on the dimension of the spacetime and boost parameter $[15,16]$. Comprehensive reviews on the Gregory-Laflamme instabilities are collected in the book [17].

In this paper we focus on the black string solution in 11 dimensions and examine its instability. Especially, since the superstring theory and M-theory contain quantum corrections to the supergravity [18]-[21], it is natural to study quantum corrections to the Gregory-Laflamme instability. This is one of the main purpose of this paper, and we take into account $R^{4}$ corrections to the 11 dimensional supergravity [22, 23]. These corrections corresponds to the leading 1-loop corrections in the type IIA superstring theory, and supersymmetric completions of these terms are discussed in refs. [24]-[30]. In this paper we briefly review the structure of the $R^{4}$ corrections in M-theory, and solve equations of motion for the configurations of the black hole and the black string. We will call these solutions quantum black hole and quantum black string, respectively. See refs. [31-33] for the related works on this topic.

Another important task of this paper is to consider the Lorentz boost of the quantum black string. In the case of classical black string, it is known that its instability highly depends on the boost parameter [16]. Similarly we will see that the thermodynamics of the quantum black string changes and the its transition point is also modified due to the boost parameter. We also investigate the near horizon limit of the boosted quantum black string, which corresponds to that of the nonextremal quantum black 0-brane.

Organization of this paper is as follows. In section 2, we review the higher derivative corrections in M-theory, and derive equations of motion. We consider the quantum corrections to the black hole and the black string and discuss the instability from entropic arguments. In section 3, we boost the quantum black hole and black string, and examine the Gregory-Laflamme instability. In section 4, the near horizon limit of the quantum black string is investigated. Section 5 is devoted to the summary of this paper and future works. Technical calculations on both quantum black hole and black string are explicitly shown in appendices $\mathrm{A}$ and $\mathrm{B}$.

\section{Quantum black hole and black string in M-theory}

\subsection{Brief review of higher derivative corrections in M-theory}

M-theory is defined as a strong coupling limit of type IIA superstring theory, and it is well approximated by 11 dimensional supergravity in the low energy limit. Classical solutions 
of 11 dimensional supergravity play important roles to reveal the structure of the M-theory. In this section, we consider black hole and black string solutions in 11 dimensions. These are quite simple and it is possible to discuss leading quantum corrections to them.

It is known that the leading correction comes from $R^{4}$ terms $[22,23]$. The bosonic part of the M-theory effective action which is relevant to the graviton is given by

$$
\begin{aligned}
& S_{11}=\frac{1}{2 \kappa_{11}^{2}} \int d^{11} x e\left\{R+\gamma\left(t_{8} t_{8} R^{4}-\frac{1}{4 !} \epsilon_{11} \epsilon_{11} R^{4}\right)\right\} \\
&=\frac{1}{2 \kappa_{11}^{2}} \int d^{11} x e\left\{R+24 \gamma\left(R_{a b c d} R_{a b c d} R_{e f g h} R_{e f g h}-64 R_{a b c d} R_{a e f g} R_{b c d h} R_{e f g h}\right.\right. \\
&+ 2 R_{a b c d} R_{a b e f} R_{c d g h} R_{e f g h}+16 R_{a c b d} R_{a e b f} R_{c g d h} R_{e g f h} \\
&\left.\left.-16 R_{a b c d} R_{a e f g} R_{b e f h} R_{c d g h}-16 R_{a b c d} R_{a e f g} R_{b f e h} R_{c d g h}\right)\right\},
\end{aligned}
$$

where $a, b, c, \cdots=0,1, \cdots, 10$ are local Lorentz indices. In this action, the expansion parameter is expressed in terms of the 11 dimensional Planck length as

$$
\gamma=\frac{\pi^{2} \ell_{\mathrm{p}}^{6}}{2^{11} 3^{2}}
$$

In eq. (2.1) pairs of indices are lowered for simplicity, but of course they should be contracted by the flat metric. Note that the above action preserves local supersymmetry, and fermionic terms are given in refs. [27]-[30]. Note also that $\gamma \sim g_{\mathrm{s}}^{2} \ell_{\mathrm{s}}^{6}$, so if we reduce the effective action (2.1) into 10 dimensions, it corresponds to 1-loop quantum correction to the type IIA supergravity.

By varying the effective action (2.1) with respect to the vielbein, we obtain equations of motion,

$$
E_{i j} \equiv R_{i j}-\frac{1}{2} \eta_{i j} R+\gamma\left\{-\frac{1}{2} \eta_{i j}\left(t_{8} t_{8} R^{4}-\frac{1}{4 !} \epsilon_{11} \epsilon_{11} R^{4}\right)+R_{a b c i} X_{j}^{a b c}-2 D_{(a} D_{b)} X^{a}{ }_{i j}^{b}\right\}=0
$$

up to the linear order of $\gamma$. Here $D_{a}$ is a covariant derivative for local Lorentz indices and $X_{a b c d}$ is defined as

$$
\begin{aligned}
X_{a b c d}= & \frac{1}{2}\left(X_{[a b][c d]}^{\prime}+X_{[c d][a b]}^{\prime}\right), \\
X_{a b c d}^{\prime}= & 96\left(R_{a b c d} R_{e f g h} R_{e f g h}-16 R_{a b c e} R_{d f g h} R_{e f g h}+2 R_{a b e f} R_{c d g h} R_{e f g h}+16 R_{a e c g} R_{b f d h} R_{e f g h}\right. \\
\quad & \left.\quad-16 R_{a b e g} R_{c f e h} R_{d f g h}-16 R_{e f a g} R_{e f c h} R_{g b h d}+8 R_{a b e f} R_{c e g h} R_{d f g h}\right) .
\end{aligned}
$$

It is not obvious but possible to check that $R_{a b c i} X^{a b c}{ }_{j}=R_{a b c j} X^{a b c}{ }_{i}$, hence $E_{i j}$ is a symmetric tensor. The explicit derivation of eq. (2.3) can be found in ref. [34].

\subsection{Quantum black hole}

First we briefly review Schwarzschild black hole solution in 11 dimensional supergravity. The metric of the black hole is given by

$$
d s_{\mathrm{h}}^{2}=-A d t^{2}+A^{-1} d r^{2}+r^{2} d \Omega_{9}^{2}, \quad A=1-\frac{r_{\mathrm{h}}^{8}}{r^{8}},
$$


and the event horizon $r_{\text {horizon }}$ is located at $r_{\text {horizon }}=r_{\mathrm{h}}$. From standard calculations, ADM mass and entropy of the black hole are evaluated as

$$
M_{\mathrm{h}}=\frac{9 V_{S^{9}} r_{\mathrm{h}}^{8}}{2 \kappa_{11}^{2}}, \quad S_{\mathrm{h}}=\frac{4 \pi V_{S^{9}} r_{\mathrm{h}}^{9}}{2 \kappa_{11}^{2}} .
$$

Here $2 \kappa_{11}^{2}=(2 \pi)^{8} \ell_{\mathrm{p}}^{9}$ and $\ell_{\mathrm{p}}$ is the Planck length in 11 dimensions. ${ }^{1} V_{S^{9}}=\frac{\pi^{5}}{12}$ is the volume of the 9 dimensional unit sphere.

Next let us take into account the quantum correction to the black hole by solving the eq. (2.3) up to the linear order of $\gamma$. The leading part of the metric (2.5) itself is not a solution of the eq. (2.3), so we should relax the ansatz. Most general static ansatz with $\mathrm{SO}(10)$ rotation symmetry is given by

$$
\begin{aligned}
d s_{\mathrm{h}}^{2} & =-B_{1}^{-1} A_{1} d t^{2}+A_{1}^{-1} d r^{2}+r^{2} d \Omega_{9}^{2}, \\
A_{1} & =1-\frac{r_{\mathrm{h}}^{8}}{r^{8}}+\frac{\gamma}{r_{\mathrm{h}}^{6}} a_{1}\left(\frac{r}{r_{\mathrm{h}}}\right), \quad B_{1}=1+\frac{\gamma}{r_{\mathrm{h}}^{6}} b_{1}\left(\frac{r}{r_{\mathrm{h}}}\right) .
\end{aligned}
$$

In order to make the equations of motion simple, we introduce following dimensionless coordinates, $\tau=\frac{t}{r_{\mathrm{h}}}, x=\frac{r}{r_{\mathrm{h}}}$, and insert the ansatz (2.7) into the eq. (2.3). Then the equations of motion become

$$
\begin{aligned}
E_{1}= & -x^{39} a_{1}^{\prime}-8 x^{38} a_{1}-9299558400 x^{8}+10492093440=0, \\
E_{2}= & x^{39} a_{1}^{\prime}+8 x^{38} a_{1}+x^{31}\left(1-x^{8}\right) b_{1}^{\prime}-312729600 x^{8}-879805440=0, \\
E_{3}= & x^{40} a_{1}^{\prime \prime}+16 x^{39} a_{1}^{\prime}+56 x^{38} a_{1}+x^{32}\left(1-x^{8}\right) b_{1}^{\prime \prime}-4 x^{31}\left(1+2 x^{8}\right) b_{1}^{\prime} \\
& +7192780800 x^{8}-11175183360=0,
\end{aligned}
$$

where the prime represents the derivative with respect to $x$. By solving $E_{1}=E_{2}=0$ with requiring asymptotic flatness, we obtain

$$
\begin{aligned}
& a_{1}(x)=-\frac{349736448}{x^{38}}+\frac{422707200}{x^{30}}+\frac{c_{\mathrm{h}}}{x^{8}}, \\
& b_{1}(x)=\frac{320409600}{x^{30}},
\end{aligned}
$$

where $c_{\mathrm{h}}$ is an integral constant. From this we see that the quantum corrections become important when $r<r_{\mathrm{h}}$ or $1 \ll \frac{\gamma}{r_{\mathrm{h}}^{6}}$. Notice that $c_{\mathrm{h}}$ can be absorbed by the redefinition of $r_{\mathrm{h}}$ like

$$
r_{\mathrm{h}}^{8}-\gamma c_{\mathrm{h}} r_{\mathrm{h}}^{2} \rightarrow r_{\mathrm{h}}^{8},
$$

up to linear order of $\gamma$. So physical quantities do not depend on $c_{\mathrm{h}}$ at this order. ${ }^{2}$ The remaining equation $E_{3}=0$ is trivially satisfied by inserting eq. (2.9). The plots of $A_{1}(x)$ are shown in appendix A.2.

\footnotetext{
${ }^{1}$ By using string length $\ell_{\mathrm{s}}$ and string coupling constant $g_{\mathrm{s}}$, the Planck length is expressed as $\ell_{\mathrm{p}}=\ell_{\mathrm{s}} g_{\mathrm{s}}^{1 / 3}$. ${ }^{2} r_{\mathrm{h}}^{8}-\gamma c_{\mathrm{h}} r_{\mathrm{h}}^{2}>0$ is required since the mass $(2.14)$ should be positive.
} 
Now we call the metric of eq. (2.7) with eq. (2.9) the quantum black hole. Let us investigate the thermodynamics of the quantum black hole. The event horizon is located at $r_{\text {horizon }}=r_{\mathrm{h}}-\frac{\gamma}{8 r_{\mathrm{h}}^{5}} a_{1}(1)$ up to the linear order of $\gamma$, and the temperature is given by

$$
\begin{aligned}
T_{\mathrm{h}} & =\left.\frac{1}{4 \pi} B_{1}^{-\frac{1}{2}} \frac{d A_{1}}{d r}\right|_{r_{\text {horizon }}} \\
& =\frac{2}{\pi r_{\mathrm{h}}}\left\{1+\gamma\left(\frac{9}{8} a_{1}(1)+\frac{1}{8} a_{1}^{\prime}(1)-\frac{1}{2} b_{1}(1)\right) \frac{1}{r_{\mathrm{h}}^{6}}\right\} \equiv \frac{2}{\pi} \bar{T}_{\mathrm{h}} .
\end{aligned}
$$

By solving the above equation inversely, $r_{\mathrm{h}}$ is expressed as

$$
r_{\mathrm{h}}=\frac{1}{\bar{T}_{\mathrm{h}}}\left\{1+\gamma\left(\frac{9}{8} a_{1}(1)+\frac{1}{8} a_{1}^{\prime}(1)-\frac{1}{2} b_{1}(1)\right) \bar{T}_{\mathrm{h}}^{6}\right\}
$$

and from this relation, physical quantities can be expressed in terms of the temperature up to the linear order of $\gamma$. For instance, the location of the event horizon is evaluated as

$$
\begin{aligned}
r_{\text {horizon }} & =\frac{1}{\bar{T}_{\mathrm{h}}}\left\{1+\gamma\left(a_{1}(1)+\frac{1}{8} a_{1}^{\prime}(1)-\frac{1}{2} b_{1}(1)\right) \bar{T}_{\mathrm{h}}^{6}\right\} \\
& =\frac{1}{\bar{T}_{\mathrm{h}}}\left(1-11137920 \gamma \bar{T}_{\mathrm{h}}^{6}\right) .
\end{aligned}
$$

This reveals that the position of the event horizon slightly moves inward due to the quantum correction, and its value does not depend on $c_{\mathrm{h}}$.

The ADM mass $M_{\mathrm{h}}$ is calculated as

$$
\begin{aligned}
\frac{2 \kappa_{11}^{2}}{9 V_{S^{9}}} M_{\mathrm{h}} & =r_{\mathrm{h}}^{8}\left(1-\gamma \frac{c_{\mathrm{h}}}{r_{\mathrm{h}}^{6}}\right) \\
& =\frac{1}{\bar{T}_{\mathrm{h}}^{8}}\left\{1+\gamma\left(9 a_{1}(1)+a_{1}^{\prime}(1)-4 b_{1}(1)-c_{\mathrm{h}}\right) \bar{T}_{\mathrm{h}}^{6}\right\}, \\
& =\frac{1}{\bar{T}_{\mathrm{h}}^{8}}\left(1-16132608 \gamma \bar{T}_{\mathrm{h}}^{6}\right) \equiv \bar{M}_{\mathrm{h}} .
\end{aligned}
$$

Note that although the effective action (2.1) contains the higher derivative terms, the expression of the ADM mass formula does not [35]. The above correction just enters through $\frac{c_{\mathrm{h}}}{x^{8}}$ term in $a_{1}(x)$ and $r_{\mathrm{h}}$ in eq. (2.12). On the other hand, the area law of the entropy is modified by the higher derivative corrections [36, 37], and the black hole entropy $S_{\mathrm{h}}$ is given by

$$
\begin{aligned}
\frac{2 \kappa_{11}^{2}}{4 \pi V_{S^{9}}} S_{\mathrm{h}} & =r_{\text {horizon }}^{9}\left(1-\left.2 \gamma X_{0101}\right|_{x=1}\right) \\
& =\frac{1}{\bar{T}_{\mathrm{h}}^{9}}\left(1-12099456 \gamma \bar{T}_{\mathrm{h}}^{6}\right) \\
& =\bar{M}_{\mathrm{h}}^{9 / 8}\left(1+6049728 \gamma \bar{M}_{\mathrm{h}}^{-3 / 4}\right) .
\end{aligned}
$$

As explained before, $r_{\text {horizon }}, M_{\mathrm{h}}$ and $S_{\mathrm{h}}$ are written in terms of $T_{\mathrm{h}}$, and do not depend on the unknown constant $c_{\mathrm{h}}$. It is easy to see that the first law $d M_{\mathrm{h}}=T_{\mathrm{h}} d S_{\mathrm{h}}$ holds up to the linear order of $\gamma$. 


\subsection{Quantum black string}

In this section, we consider a black string solution which is constructed by aligning 10 dimensional black hole along the 11th direction. The 11th direction is compactified on a circle and its radius is given by $R_{11}=\ell_{\mathrm{s}} g_{\mathrm{s}}$. In 11 dimensional supergravity, the black string solution is simply given by

$$
d s_{\mathrm{s}}^{2}=-F d t^{2}+F^{-1} d r^{2}+r^{2} d \Omega_{8}^{2}+d z^{2}, \quad F=1-\frac{r_{\mathrm{s}}^{7}}{r^{7}} .
$$

The ADM mass and the entropy are calculated as

$$
M_{\mathrm{s}}=\frac{8 V_{S^{8}} r_{\mathrm{s}}^{7}}{2 \kappa_{10}^{2}}, \quad S_{\mathrm{s}}=\frac{4 \pi V_{S^{8}} r_{\mathrm{s}}^{8}}{2 \kappa_{10}^{2}},
$$

where $V_{S^{8}}=\frac{2^{5} \pi^{4}}{105}$ is the volume of the 8 dimensional unit sphere, and $2 \kappa_{10}^{2}=2 \kappa_{11}^{2} /\left(2 \pi R_{11}\right)$. These expressions simply show that the 11 dimensional black string corresponds to the 10 dimensional black hole after the dimensional reduction.

Now we take into account the quantum corrections to the black string. Since the metric does not satisfy the equations of motion (2.3), we relax the ansatz as follows.

$$
\begin{aligned}
d s_{\mathrm{s}}^{2} & =-G_{1}^{-1} F_{1} d t^{2}+F_{1}^{-1} d r^{2}+r^{2} d \Omega_{8}^{2}+G_{2} d z^{2}, \\
F_{1} & =1-\frac{r_{\mathrm{s}}^{7}}{r^{7}}+\frac{\gamma}{r_{\mathrm{s}}^{6}} f_{1}\left(\frac{r}{r_{\mathrm{s}}}\right), \quad G_{i}=1+\frac{\gamma}{r_{\mathrm{s}}^{6}} g_{i}\left(\frac{r}{r_{\mathrm{s}}}\right) .
\end{aligned}
$$

This is the most general ansatz which preserves $\mathrm{SO}(9)$ rotation symmetry. In order to make the equations of motion simple, we introduce dimensionless coordinates, $\tau=\frac{t}{r_{\mathrm{h}}}, x=$ $\frac{r}{r_{\mathrm{h}}}, y=\frac{z}{r_{\mathrm{h}}}$, and insert the ansatz (2.18) into eq. (2.3). Then the equations of motion become

$$
\begin{aligned}
E_{1}= & -16 x^{35} f_{1}^{\prime}-112 x^{34} f_{1}+2 x^{29}\left(1-x^{7}\right) g_{2}^{\prime \prime}+x^{28}\left(9-16 x^{7}\right) g_{2}^{\prime} \\
& -63402393600 x^{7}+71292856320=0, \\
E_{2}= & 16 x^{35} f_{1}^{\prime}+112 x^{34} f_{1}+16 x^{28}\left(1-x^{7}\right) g_{1}^{\prime}-x^{28}\left(9-16 x^{7}\right) g_{2}^{\prime} \\
& -2159861760 x^{7}-5730600960=0, \\
E_{3}= & 2 x^{36} f_{1}^{\prime \prime}+28 x^{35} f_{1}^{\prime}+84 x^{34} f_{1}+2 x^{29}\left(1-x^{7}\right) g_{1}^{\prime \prime}-7 x^{28}\left(1+2 x^{7}\right) g_{1}^{\prime} \\
& -2 x^{29}\left(1-x^{7}\right) g_{2}^{\prime \prime}+14 x^{35} g_{2}^{\prime}+5669637120 x^{7}-8626383360=0, \\
E_{4}= & 2 x^{36} f_{1}^{\prime \prime}+32 x^{35} f_{1}^{\prime}+112 x^{34} f_{1}+2 x^{29}\left(1-x^{7}\right) g_{1}^{\prime \prime}-x^{28}\left(5+16 x^{7}\right) g_{1}^{\prime} \\
& -1062512640=0 .
\end{aligned}
$$

By solving the equations of motion (2.3) up to the linear order of $\gamma$, the functions $f_{1}$ and 
$g_{i}(i=1,2)$ are explicitly solved as

$$
\begin{aligned}
& f_{1}(x)=-\frac{1208170880}{9 x^{34}}+\frac{161405664}{x^{27}}+\frac{5738880}{13 x^{20}}+\frac{956480}{x^{13}}+\frac{c_{\mathrm{s}}}{x^{7}}+\frac{819840}{x^{7}} I(x), \\
& g_{1}(x)=\frac{1035722240}{9 x^{27}}+\frac{1721664}{x^{20}}+\frac{22955520}{13 x^{13}}+\frac{1912960}{x^{6}}-1639680 \frac{x-1}{x^{7}-1}+234240 I(x), \\
& g_{2}(x)=-\frac{94330880}{9 x^{27}}+\frac{655872}{x^{20}}+\frac{13117440}{13 x^{13}}+\frac{2186240}{x^{6}}+1873920 I(x) .
\end{aligned}
$$

Here $c_{\mathrm{s}}$ is an integral constant. The details of the derivation and an explicit form of $I(x)$ can be found in appendix B.2. There other integral constants are set to be zero so that the geometry becomes asymptotically flat. Notice that $c_{\mathrm{S}}$ can be absorbed by the redefinition of $r_{\mathrm{s}}$ up to the linear order of $\gamma$, so physical quantities do not depend on $c_{\mathrm{s}}$.

Let us investigate the thermodynamics of the quantum black string. The event horizon is located at $r_{\text {horizon }}=r_{\mathrm{s}}-\frac{\gamma}{7 r_{\mathrm{s}}^{5}} f_{1}(1)$, and the temperature is evaluated as

$$
\begin{aligned}
T_{\mathrm{s}} & =\left.\frac{1}{4 \pi} G_{1}^{-\frac{1}{2}} \frac{d F_{1}}{d r}\right|_{r_{\text {horizon }}} \\
& =\frac{7}{4 \pi r_{\mathrm{s}}}\left\{1+\gamma\left(\frac{8}{7} f_{1}(1)+\frac{1}{7} f_{1}^{\prime}(1)-\frac{1}{2} g_{1}(1)\right) \frac{1}{r_{\mathrm{s}}^{6}}\right\} \equiv \frac{7}{4 \pi} \bar{T}_{\mathrm{s}} .
\end{aligned}
$$

By solving this inversely, $r_{\mathrm{s}}$ is expressed as

$$
r_{\mathrm{s}}=\frac{1}{\bar{T}_{\mathrm{s}}}\left\{1+\gamma\left(\frac{8}{7} f_{1}(1)+\frac{1}{7} f_{1}^{\prime}(1)-\frac{1}{2} g_{1}(1)\right) \bar{T}_{\mathrm{s}}^{6}\right\}
$$

From this relation, $r_{\mathrm{s}}$ is replaced with the temperature when we calculate physical quantities up to the linear order of $\gamma$. For example, the location of the event horizon is given by

$$
\begin{aligned}
r_{\text {horizon }} & =\frac{1}{\bar{T}_{\mathrm{s}}}\left\{1+\gamma\left(f_{1}(1)+\frac{1}{7} f_{1}^{\prime}(1)-\frac{1}{2} g_{1}(1)\right) \bar{T}_{\mathrm{s}}^{6}\right\} \\
& =\frac{1}{\bar{T}_{\mathrm{s}}}\left\{1-\gamma\left(\frac{587024224}{117}+117120 I(1)\right) \bar{T}_{\mathrm{s}}^{6}\right\} .
\end{aligned}
$$

The location of the horizon moves inward and does not depends on $c_{\mathrm{s}}$, just like the case of the quantum black hole.

The ADM mass of the black string $M_{\mathrm{S}}$ is evaluated as

$$
\begin{aligned}
\frac{2 \kappa_{10}^{2}}{8 V_{S^{8}}} M_{\mathrm{s}} & =r_{\mathrm{s}}^{7}\left(1+\gamma \frac{1639680-c_{\mathrm{s}}}{r_{\mathrm{s}}^{6}}\right) \\
& =\frac{1}{\bar{T}_{\mathrm{s}}^{7}}\left\{1+\gamma\left(1639680+8 f_{1}(1)+f_{1}^{\prime}(1)-\frac{7}{2} g_{1}(1)-c_{\mathrm{s}}\right) \bar{T}_{\mathrm{s}}^{6}\right\} \\
& =\frac{1}{\bar{T}_{\mathrm{s}}^{7}}\left(1-4919040 \gamma \bar{T}_{\mathrm{s}}^{6}\right) \equiv \bar{M}_{\mathrm{s}} .
\end{aligned}
$$

Note that we used $2 \kappa_{10}^{2}=2 \kappa_{11}^{2} /\left(2 \pi R_{11}\right)$. The mass formula itself is not modified, but the corrections in the first line enter through $x^{-7}$ terms in $f_{1}(x)$ and $g_{2}(x)$. On the other hand, 
the entropy $S_{\mathrm{s}}$ of the quantum black string receives the higher derivative corrections, and is calculated as

$$
\begin{aligned}
\frac{2 \kappa_{10}^{2}}{4 \pi V_{S^{8}}} S_{\mathrm{s}} & =r_{\text {horizon }}^{8} G_{2}(1)^{1 / 2}\left(1-\left.2 \gamma X_{0101}\right|_{x=1}\right) \\
& =\frac{1}{\bar{T}_{\mathrm{s}}^{8}}\left(1-2810880 \gamma \bar{T}_{\mathrm{s}}^{6}\right) \\
& =\bar{M}_{\mathrm{s}}^{8 / 7}\left(1+2810880 \gamma \bar{M}_{\mathrm{s}}^{-6 / 7}\right) .
\end{aligned}
$$

The entropy formula is affected by the higher derivative corrections. Note that $r_{\text {horizon }}$, $M_{\mathrm{S}}$ and $S_{\mathrm{s}}$ do not depend on $c_{\mathrm{s}}$, and the first law of the thermodynamics $d M_{\mathrm{s}}=T_{\mathrm{s}} d S_{\mathrm{s}}$ is satisfied up to the linear of $\gamma$.

\subsection{Gregory-Laflamme instability of the quantum black string}

It is known that the black string is unstable against perturbation when the size of the compactified direction is large compared to the size of the black hole. In order to give thermodynamic argument on this instability, so called Gregory-Laflamme instability, we compare $S_{\mathrm{h}}$ with $S_{\mathrm{s}}$ for the equal mass.

In terms of a dimensionless parameter $M=\ell_{\mathrm{S}} M_{\mathrm{h}}=\ell_{\mathrm{S}} M_{\mathrm{s}}$, the entropy of the quantum black hole is given by

$$
S_{\mathrm{h}}=8 \pi^{2}\left(\frac{g_{\mathrm{s}}^{3} M^{9}}{9^{9} V_{S^{9}}}\right)^{1 / 8}\left\{1+\frac{10503}{2^{11} \pi^{4}} \frac{1}{g_{\mathrm{s}}^{4}}\left(\frac{9 V_{S^{9}} g_{\mathrm{s}}^{5}}{M}\right)^{3 / 4}\right\}
$$

and that of the quantum black string is given by

$$
S_{\mathrm{s}}=8 \pi^{2}\left(\frac{g_{\mathrm{s}}^{2} M^{8}}{8^{8} V_{S^{8}}}\right)^{1 / 7}\left\{1+\frac{305}{2^{7} \pi^{4}} \frac{1}{g_{\mathrm{s}}^{4}}\left(\frac{8 V_{S^{8}} g_{\mathrm{s}}^{5}}{M}\right)^{6 / 7}\right\} .
$$

Here we used $2 \kappa_{11}^{2}=2 \kappa_{10}^{2}\left(2 \pi R_{11}\right)=(2 \pi)^{8} \ell_{\mathrm{s}}^{9} g_{\mathrm{s}}^{3}$. The instability of the black string is estimated by comparing these two entropies. From eqs. (2.26) and (2.27), up to the linear order of $\gamma$, the ratio of the entropies is evaluated as

$$
\begin{aligned}
\frac{S_{\mathrm{h}}}{S_{\mathrm{s}}} & =L\left\{1+\frac{10503}{2^{11} \pi^{4}}\left(\frac{9^{8} V_{S^{9}}}{8^{8} V_{S^{8}}}\right)^{6} \frac{L^{42}}{g_{\mathrm{s}}^{4}}-\frac{305}{2^{7} \pi^{4}}\left(\frac{9^{9} V_{S^{9}}}{8^{9} V_{S^{8}}}\right)^{6} \frac{L^{48}}{g_{\mathrm{s}}^{4}}\right\} \\
& \sim L+6.04 \frac{L^{43}}{g_{\mathrm{s}}^{4}}-5.69 \frac{L^{49}}{g_{\mathrm{s}}^{4}},
\end{aligned}
$$

where we defined

$$
L \equiv \frac{\left(8^{8} V_{S^{8}}\right)^{1 / 7}}{\left(9^{9} V_{S^{9}}\right)^{1 / 8}}\left(\frac{g_{\mathrm{s}}^{5}}{M}\right)^{1 / 56} \sim 0.984\left(\frac{g_{\mathrm{s}}^{5}}{M}\right)^{1 / 56} .
$$

In the classical supergravity limit, if we increase $L$ from zero, the Gregory-Laflamme transition from the black string to the black hole occurs at $L=1$. By taking into account the quantum effect, the transition point also depends on the value of $g_{\mathrm{s}}$ like

$$
L=1-\frac{0.350}{g_{\mathrm{s}}^{4}}
$$

The plot of $S_{\mathrm{h}} / S_{\mathrm{s}}$ is drawn in figure 1 . 
Finally let us clarify the validity of the approximation (2.28) qualitatively. So far we have analyzed the black hole and black string solutions by considering quantum corrections (2.1) in 11 dimensions. Since the 11th direction is compactified, the effective action corresponds to the type IIA superstring theory and expanded by $g_{\mathrm{s}}^{2} e^{2 \phi}$ and $\ell_{\mathrm{s}}^{2}$. Then we can examine the validity of eq. (2.28) by estimating the other higher derivative terms in the type IIA superstring theory, which can be done by evaluating the dilaton $\phi$ and the Riemann tensor $R_{a b c d}$ in 10 dimensions from eq. (2.16). From the standard relation $d s_{\mathrm{s}}^{2}=e^{-\frac{2}{3} \phi} d s_{10}^{2}+e^{\frac{4}{3} \phi} d z^{2}$, we obtain

$$
g_{\mathrm{s}} e^{\phi}=g_{\mathrm{s}}, \quad \ell_{\mathrm{s}}^{2} R_{a b c d} \sim \frac{\ell_{\mathrm{s}}^{2}}{r_{\mathrm{s}}^{2}} \sim \ell_{\mathrm{s}}^{2} \bar{M}_{\mathrm{s}}^{-2 / 7},
$$

at the horizon. Thus a generic term is estimated as

$$
\left(g_{\mathrm{s}}^{2} e^{2 \phi}\right)^{n}\left(\ell_{\mathrm{s}}^{2} R_{a b c d}\right)^{m} \sim g_{\mathrm{s}}^{2 n} \ell_{\mathrm{s}}^{2 m} \bar{M}_{\mathrm{s}}^{-2 m / 7} \sim g_{\mathrm{s}}^{2 n-4 m / 7} M^{-2 m / 7} \sim g_{\mathrm{s}}^{2 n-2 m} L^{16 m} .
$$

It is known that the leading tree, 1-loop and $n(\geq 2)$-loop corrections in the type IIA superstring theory become $(n, m)=(0,3),(1,3),(n, n+3)$, respectively [38]. So the estimations of leading tree, 1-loop and $n(\geq 2)$-loop are given by $g_{\mathrm{s}}^{-6} L^{48}, g_{\mathrm{s}}^{-4} L^{48}$ and $g_{\mathrm{s}}^{-6} L^{16 n+48}$, respectively. In a similar way, from the dimensional analysis, the quantum corrections for the quantum black hole are estimated like $g_{\mathrm{s}}^{2 n} \ell_{\mathrm{s}}^{2 m} \bar{M}_{\mathrm{h}}^{-m / 4} \sim g_{\mathrm{s}}^{2 n-3 m / 4} M^{-m / 4} \sim g_{\mathrm{s}}^{2 n-2 m} L^{14 m}$.

From the above discussions, we expect that the ratio of the entropies (2.28) is reliable when $L \leq 1$ and $1 \ll g_{\mathrm{s}}^{2}$, if each term has a coefficient of order unity. It is interesting to note that, around the transition point $L \sim 1$, the supergravity approximation is valid when $g_{\mathrm{s}}$ goes to infinity, and quantum effects become quite important around $g_{\mathrm{s}} \sim 1$.

\section{Boosted quantum black hole and black string in M-theory}

\subsection{Boosted quantum black hole}

From an observer at infinity, the quantum black hole is localized at the origin of 10 dimensional space. Now let us Lorentz boost the quantum black hole along the 11th direction. Then the observer see that the quantum black hole carries a momentum along the 11th direction.

First the mass $M_{\mathrm{h}}$ and the momentum $Q_{\mathrm{h}}$ of the boosted quantum black hole are simply given by

$$
\begin{aligned}
& \frac{2 \kappa_{11}^{2}}{9 V_{S^{9}}} M_{\mathrm{h}}=r_{\mathrm{h}}^{8} \cosh \eta\left(1-\gamma \frac{c_{\mathrm{h}}}{r_{\mathrm{h}}^{6}}\right) \equiv \bar{M}_{\mathrm{h}}, \\
& \frac{2 \kappa_{11}^{2}}{9 V_{S^{9}}} Q_{\mathrm{h}}=r_{\mathrm{h}}^{8} \sinh \eta\left(1-\gamma \frac{c_{\mathrm{h}}}{r_{\mathrm{h}}^{6}}\right) \equiv \bar{Q}_{\mathrm{h}},
\end{aligned}
$$

where $\eta$ is a boost parameter. ${ }^{3}$ By combining these two equations, the parameter of the quantum black hole is expressed as

$$
r_{\mathrm{h}}^{8}\left(1-\gamma \frac{c_{\mathrm{h}}}{r_{\mathrm{h}}^{6}}\right)=\bar{M}_{\mathrm{h}}\left(1-\frac{\bar{Q}_{\mathrm{h}}^{2}}{\bar{M}_{\mathrm{h}}^{2}}\right)^{1 / 2} .
$$

\footnotetext{
${ }^{3}$ We assigned the same symbol $M_{\mathrm{h}}$ as in the section 2.2 , since it would not cause any confusion.
} 


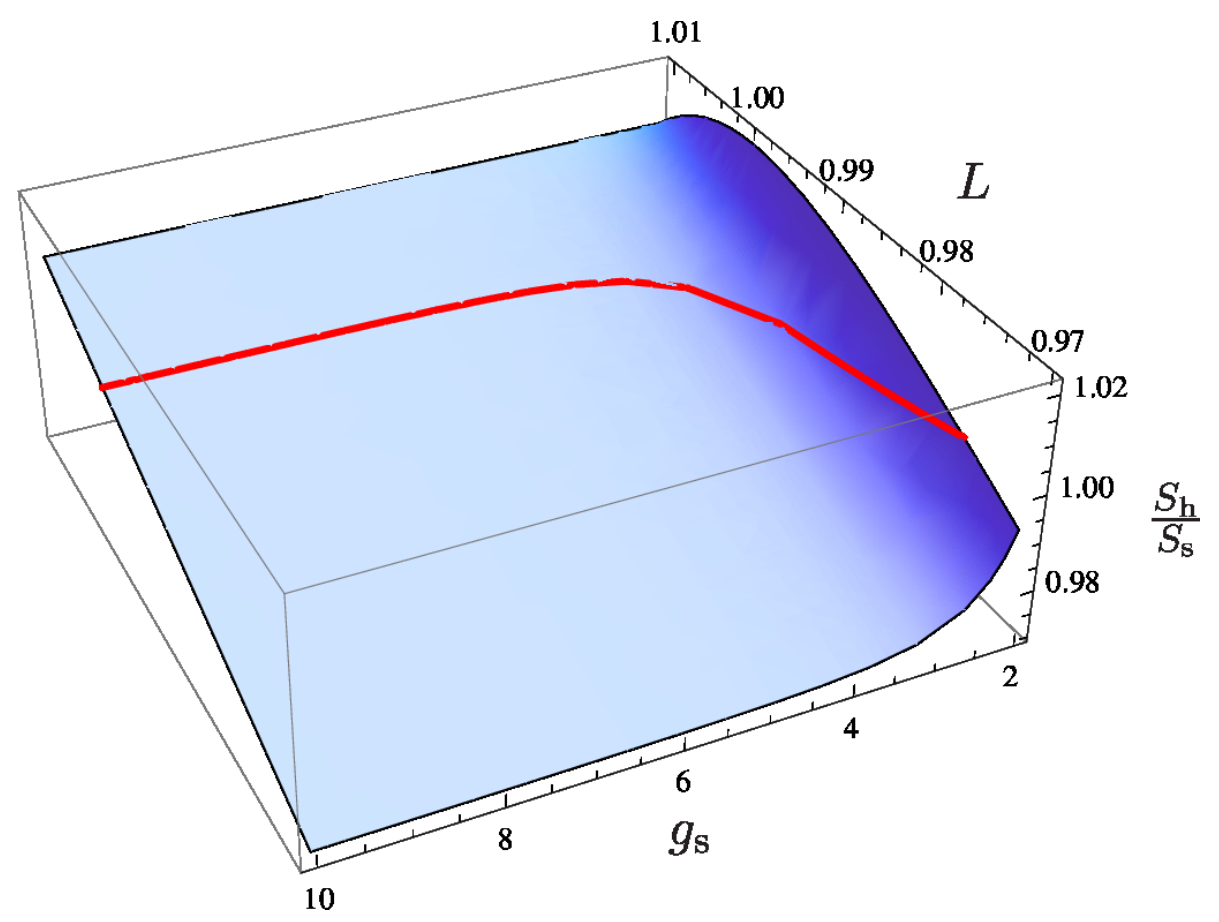

Figure 1. Plot of eq. (2.28) which is valid when $L \leq 1$. A red line correspond to $S_{\mathrm{h}}=S_{\mathrm{s}}$.

Next we examine the entropy of the boosted quantum black hole. It is known that the volume of the event horizon is invariant under the Lorentz boost [39]. The higher derivative term $X_{0101}=\frac{1}{4} N^{a b} N^{c d} X_{a b c d}$ is invariant as well. Therefore the entropy formula is entirely invariant under the Lorentz boost and given by eq. (2.15) by replacing $\bar{M}_{\mathrm{h}}$ with the right hand side of eq. (3.2).

$$
\frac{2 \kappa_{11}^{2}}{4 \pi V_{S^{9}}} S_{\mathrm{h}}=\bar{M}_{\mathrm{h}}^{9 / 8}\left(1-\frac{\bar{Q}_{\mathrm{h}}^{2}}{\bar{M}_{\mathrm{h}}^{2}}\right)^{9 / 16}\left\{1+6049728 \gamma \bar{M}_{\mathrm{h}}^{-3 / 4}\left(1-\frac{\bar{Q}_{\mathrm{h}}^{2}}{\bar{M}_{\mathrm{h}}^{2}}\right)^{-3 / 8}\right\} .
$$

Of course, eq. (2.15) is recovered when $\bar{Q}_{\mathrm{h}}=0$.

\subsection{Boosted quantum black string}

Let us briefly review the Lorentz boost of the black string (2.16) along 11th direction. The boost is executed on $(t, z)$-plane and the metric becomes

$$
\begin{aligned}
d s_{\mathrm{s}}^{2} & =-F(\cosh \beta d t+\sinh \beta d z)^{2}+(\sinh \beta d t+\cosh \beta d z)^{2}+F^{-1} d r^{2}+r^{2} d \Omega_{8}^{2} \\
& =-H^{-1} F d t^{2}+H\left(d z+\left(1-H^{-1}\right) \frac{\cosh \beta}{\sinh \beta} d t\right)^{2}+F^{-1} d r^{2}+r^{2} d \Omega_{8}^{2}
\end{aligned}
$$

where $\beta$ is a boost parameter. Here $H$ is defined as

$$
H=1+\frac{r_{\mathrm{s}}^{7} \sinh ^{2} \beta}{r^{7}},
$$


and $F$ is defined in eq. (2.16). This geometry corresponds to the nonextremal M-wave solution in 11 dimensional supergravity, whose mass and momentum along the 11th direction are expressed by two parameters $r_{\mathrm{s}}$ and $\beta$. We also use $\alpha^{7} \equiv 1 / \sinh ^{2} \beta$ for the boost parameter. ${ }^{4}$ Note that the nonextremal M-wave solution is identified with the nonextremal black 0-brane solution in 10 dimensions.

In the same way, it is possible to boost the quantum black string solution (2.18) along the 11th direction, and the metric becomes

$$
\begin{aligned}
d s_{\mathrm{s}}^{2} & =-G_{1}^{-1} F_{1}(\cosh \beta d t+\sinh \beta d z)^{2}+G_{2}(\sinh \beta d t+\cosh \beta d z)^{2}+F_{1}^{-1} d r^{2}+r^{2} d \Omega_{8}^{2} \\
& =-H_{1}^{-1} F_{1} d t^{2}+H_{2}\left(d z+\left(1-H_{2}^{-\frac{1}{2}} H_{3}^{-\frac{1}{2}}\right) \frac{\cosh \beta}{\sinh \beta} d t\right)^{2}+F_{1}^{-1} d r^{2}+r^{2} d \Omega_{8}^{2} .
\end{aligned}
$$

Here $F_{1}$ is defined in eq. (2.18), and $H_{i}(i=1,2,3)$ are expressed in terms of $F_{1}, G_{1}$ and $G_{2}$ as

$$
H_{1}=G_{1} G_{2}^{-1} H_{2}, \quad H_{2}=G_{2}+\left(G_{2}-G_{1}^{-1} F_{1}\right) \sinh ^{2} \beta, \quad H_{3}=G_{2}^{-2} H_{2} .
$$

Now we choose $r_{\mathrm{s}}$ and $\alpha$ as independent parameters. Then the above functions can be expressed up to the linear order of $\gamma$ as

$$
\begin{aligned}
& H_{1}=1+\frac{1}{\alpha^{7} x^{7}}+\frac{\gamma}{r_{\mathrm{s}}^{6} \alpha^{7}}\left\{-f_{1}(x)+\left(1+\alpha^{7}\right) g_{1}(x)+\left(1-\frac{1}{x^{7}}\right) g_{2}(x)\right\} \\
& H_{2}=1+\frac{1}{\alpha^{7} x^{7}}+\frac{\gamma}{r_{\mathrm{s}}^{6} \alpha^{7}}\left\{-f_{1}(x)+\left(1-\frac{1}{x^{7}}\right) g_{1}(x)+\left(1+\alpha^{7}\right) g_{2}(x)\right\} \\
& H_{3}=1+\frac{1}{\alpha^{7} x^{7}}+\frac{\gamma}{r_{\mathrm{s}}^{6} \alpha^{7}}\left\{-f_{1}(x)+\left(1-\frac{1}{x^{7}}\right) g_{1}(x)+\left(1-\alpha^{7}-\frac{2}{x^{7}}\right) g_{2}(x)\right\},
\end{aligned}
$$

where $x=\frac{r}{r_{\mathrm{s}}}$. By inserting eq. (2.20) and $c_{\mathrm{s}}=3747840$ into the above, we obtain eq. (48) in ref. [35]. In that paper $c_{\mathrm{s}}=3747840$ is required so as to be consistent with the near horizon limit which will be explained in section 4. Thus the geometry (3.6) is exactly the same as that of the quantum M-wave solution in 11 dimensions. The dimensional reduction of the metric corresponds to the quantum black 0-brane solution in 10 dimensions.

Let us examine the thermodynamics of the boosted quantum black string by choosing $c_{\mathrm{s}}=3747840$. The event horizon is located at $r_{\text {horizon }}=r_{\mathrm{s}}-\frac{\gamma}{7 r_{\mathrm{s}}^{5}} f_{1}(1)$, and the temperature is evaluated as

$$
\begin{aligned}
T_{\mathrm{s}} & =\left.\frac{1}{4 \pi} H_{1}^{-1 / 2} \frac{d F_{1}}{d r}\right|_{r_{\text {horizon }}} \\
& =\frac{7}{4 \pi r_{\mathrm{s}}} \frac{\alpha^{7 / 2}}{\sqrt{1+\alpha^{7}}}\left\{1+\gamma\left(\frac{8}{7} f_{1}(1)+\frac{1}{7} f_{1}^{\prime}(1)-\frac{1}{2} g_{1}(1)\right) \frac{1}{r_{\mathrm{s}}^{6}}\right\} \\
& =\frac{7}{4 \pi r_{\mathrm{s}}} \frac{\alpha^{7 / 2}}{\sqrt{1+\alpha^{7}}}\left(1-\frac{2810880 \gamma}{7 r_{\mathrm{s}}^{6}}\right) .
\end{aligned}
$$

\footnotetext{
${ }^{4}$ These are related to $r_{ \pm}$as $r_{-}^{7}=r_{\mathrm{s}}^{7} \sinh ^{2} \beta, r_{+}^{7}=r_{-}^{7}\left(1+\alpha^{7}\right)=r_{\mathrm{s}}^{7} \cosh ^{2} \beta$.
} 
Note that $\frac{\alpha^{7 / 2}}{\sqrt{1+\alpha^{7}}}=\frac{1}{\cosh \beta}$ comes from the time dilation of the quantum black string measured by the boosted observer.

The ADM mass and the momentum of the boosted quantum black string are calculated as [35]

$$
\begin{aligned}
& \frac{2 \kappa_{10}^{2}}{8 V_{S^{8}}} M_{\mathrm{s}}=r_{\mathrm{s}}^{7}\left(1+\frac{7}{8 \alpha^{7}}-\frac{2108160 \gamma}{r_{\mathrm{s}}^{6}}\right) \equiv \bar{M}_{\mathrm{s}} \\
& \frac{2 \kappa_{10}^{2}}{8 V_{S^{8}}} Q_{\mathrm{s}}=\frac{7 r_{\mathrm{s}}^{7} \sqrt{1+\alpha^{7}}}{8 \alpha^{7}} \equiv \bar{Q}_{\mathrm{s}}
\end{aligned}
$$

The momentum does not receive any quantum correction and is equal to the charge of $N$ D0-branes. By solving eqs. (3.10) inversely, up to the linear order of $\gamma$, the parameters $\alpha^{7}$ and $r_{\mathrm{s}}^{7}$ can be expressed as

$$
\alpha^{7}=\left(\frac{7 p}{8}\right)^{2}-1, \quad r_{\mathrm{s}}^{7}=\bar{Q}_{\mathrm{s}} p\left(1-\frac{64}{49 p^{2}}\right)
$$

where

$$
\begin{aligned}
& p=p_{0}+2108160 \gamma p_{1}, \\
& p_{0}=\frac{\bar{M}_{\mathrm{s}}}{2 \bar{Q}_{\mathrm{s}}}\left(1+\sqrt{1+\frac{32 \bar{Q}_{\mathrm{s}}^{2}}{49 \bar{M}_{\mathrm{s}}^{2}}}\right), \\
& p_{1}=\bar{Q}_{\mathrm{s}}^{-6 / 7}\left(1+\frac{8}{49 p_{0}^{2}}\right)^{-1}\left\{p_{0}\left(1-\frac{64}{49 p_{0}^{2}}\right)\right\}^{1 / 7} .
\end{aligned}
$$

It is easy to see that $p_{0} \rightarrow \infty$ and $Q_{\mathrm{s}} p_{0} \rightarrow M_{\mathrm{s}}$, if we take $Q_{\mathrm{s}} \rightarrow 0$.

The entropy of the boosted quantum black string is obtained by taking into account an expansion of the proper length along the 11th direction at the event horizon. From eqs. (3.6) and (3.7), the expansion rate of the proper length at the event horizon is given by $\left.\sqrt{H_{2} / G_{2}}\right|_{\text {horizon }}=\sqrt{1+\alpha^{7}} / \alpha^{7 / 2}$. Thus, by multiplying this factor with eq. (2.25), we obtain the entropy of the boosted quantum black string like

$$
\begin{aligned}
\frac{2 \kappa_{10}^{2}}{4 \pi V_{S^{8}}} S_{\mathrm{s}} & =r_{\text {horizon }}^{8} \frac{\sqrt{1+\alpha^{7}}}{\alpha^{7 / 2}} G_{2}(1)^{1 / 2}\left(1-\left.2 \gamma X_{0101}\right|_{x=1}\right) \\
& =r_{\mathrm{s}}^{8} \frac{\sqrt{1+\alpha^{7}}}{\alpha^{7 / 2}}\left(1+\frac{2810880 \gamma}{7 r_{\mathrm{s}}^{6}}\right) \\
& =\left(\bar{Q}_{\mathrm{s}} p_{0}\right)^{8 / 7}\left(1-\frac{64}{49 p_{0}^{2}}\right)^{9 / 14}\left\{1+2810880 \gamma\left(\bar{Q}_{\mathrm{s}} p_{0}\right)^{-6 / 7}\left(1-\frac{64}{49 p_{0}^{2}}\right)^{-6 / 7}\right\}
\end{aligned}
$$

In order to derive the second line, we used $c_{\mathrm{s}}=3747840$. This satisfies the first law of the black hole thermodynamics up to the linear order of $\gamma$. 


\subsection{Gregory-Laflamme instability of the boosted quantum black string}

The Gregory-Laflamme instability of the boosted black string is well discussed in refs. [16, 39]. In this subsection, we examine the Gregory-Laflamme instability of the boosted quantum black string. In order to compare the entropy of the boosted black hole with that of the boosted quantum black string, masses and charges should be equal respectively.

Now we set $M=\ell_{\mathrm{s}} M_{\mathrm{h}}=\ell_{\mathrm{s}} M_{\mathrm{s}}$ and $Q=\ell_{\mathrm{s}} Q_{\mathrm{h}}=\ell_{\mathrm{s}} Q_{\mathrm{s}}$, and use the relation $2 \kappa_{11}^{2}=$ $2 \kappa_{10}^{2}\left(2 \pi R_{11}\right)=(2 \pi)^{8} \ell_{\mathrm{s}}^{9} g_{\mathrm{s}}^{3}$. Then the entropy of the boosted quantum black hole is expressed as

$$
S_{\mathrm{h}}=8 \pi^{2}\left(\frac{g_{\mathrm{s}}^{3} M^{9}}{9^{9} V_{S^{9}}}\right)^{1 / 8}\left(1-\frac{Q^{2}}{M^{2}}\right)^{9 / 16}\left\{1+\frac{10503}{2^{11} \pi^{4}} \frac{1}{g_{\mathrm{s}}^{4}}\left(\frac{9 V_{S^{9}} g_{\mathrm{s}}^{5}}{M}\right)^{3 / 4}\left(1-\frac{Q^{2}}{M^{2}}\right)^{-3 / 8}\right\} .
$$

This is a generalization of eq. (2.26). In a similar way, the entropy of the boosted quantum black string is given by

$$
\begin{aligned}
S_{\mathrm{s}}= & 8 \pi^{2}\left(\frac{g_{\mathrm{s}}^{2} M^{8}}{8^{8} V_{S^{8}}}\right)^{1 / 7}\left(\frac{Q p_{0}}{M}\right)^{8 / 7}\left(1-\frac{64}{49 p_{0}^{2}}\right)^{9 / 14} \\
& \times\left\{1+\frac{305}{2^{7} \pi^{4}} \frac{1}{g_{\mathrm{s}}^{4}}\left(\frac{8 V_{S^{8}} g_{\mathrm{s}}^{5}}{M}\right)^{6 / 7}\left(\frac{Q p_{0}}{M}\right)^{-6 / 7}\left(1-\frac{64}{49 p_{0}^{2}}\right)^{-6 / 7}\right\} .
\end{aligned}
$$

Here $p_{0}$ is defined in eq. (3.12) and written in terms of $\frac{Q}{M}$. This expression is a generalization of eq. (2.27), and the ration of the entropies (2.28) is also generalized as

$$
\begin{aligned}
\frac{S_{\mathrm{h}}}{S_{\mathrm{s}}} \sim & L\left(1-\frac{Q^{2}}{M^{2}}\right)^{9 / 16}\left(\frac{Q p_{0}}{M}\right)^{-8 / 7}\left(1-\frac{64}{49 p_{0}^{2}}\right)^{-9 / 14} \\
& \times\left\{1+6.04 \frac{L^{42}}{g_{\mathrm{s}}^{4}}\left(1-\frac{Q^{2}}{M^{2}}\right)^{-3 / 8}-5.69 \frac{L^{48}}{g_{\mathrm{s}}^{4}}\left(\frac{Q p_{0}}{M}\right)^{-6 / 7}\left(1-\frac{64}{49 p_{0}^{2}}\right)^{-6 / 7}\right\},
\end{aligned}
$$

where $L$ is defined by eq. (2.29). The ratio of the entropies are parametrized by $L, g_{\mathrm{s}}$ and $\frac{Q}{M}$. From this equation, we can estimate the instability of the boosted quantum black string. The plot of eq. (3.16) with $\frac{Q}{M}=0.9$ is drawn in figure 2. From the plot we see that, at transition point, the value of $L$ for the boosted quantum black string is smaller than that of the quantum black string. It is also interesting to note that there is another transition point for $g_{\mathrm{s}}<4$, though the validity of it depends on the structure of the higher derivative terms as discussed below.

Finally let us examine the validity of eq. (3.16) by estimating the other higher derivative terms. In order to do this, we simply repeat the discussions in the section 2.4. From eq. (3.4), values of the dilaton $\phi$ and the Riemann tensor $R_{a b c d}$ in 10 dimensions are estimated as

$$
\begin{aligned}
g_{\mathrm{s}} e^{\phi} & =g_{\mathrm{s}}\left(1+\frac{1}{\alpha^{7}}\right)^{3 / 4}=g_{\mathrm{s}}\left(1-\frac{64}{49 p_{0}^{2}}\right)^{-3 / 4} \\
\ell_{\mathrm{s}}^{2} R_{a b c d} & \sim \frac{\ell_{\mathrm{s}}^{2}}{r_{\mathrm{s}}^{2}} w_{a b c d}\left(p_{0}\right)\left(1+\frac{1}{\alpha^{7}}\right)^{-1 / 2} \sim \ell_{\mathrm{s}}^{2} w_{a b c d}\left(p_{0}\right)\left(\bar{Q}_{\mathrm{s}} p_{0}\right)^{-2 / 7}\left(1-\frac{64}{49 p_{0}^{2}}\right)^{3 / 14},
\end{aligned}
$$




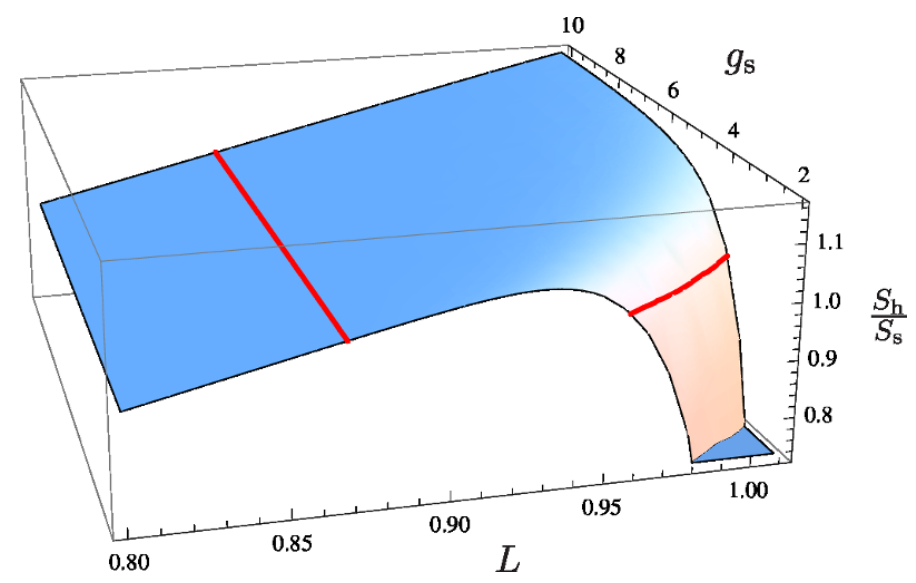

Figure 2. Plot of eq. (3.16) with $\frac{Q}{M}=0.9$. Red lines correspond to $S_{\mathrm{h}}=S_{\mathrm{s}}$.

at the horizon. Here explicit form of $w_{a b c d}\left(p_{0}\right)$ is different for each component, but becomes constant if we take $\frac{Q}{M} \rightarrow 1$. From the above a generic term is estimated as

$$
\begin{aligned}
\left(g_{\mathrm{s}}^{2} e^{2 \phi}\right)^{n}\left(\ell_{\mathrm{s}}^{2} R_{a b c d}\right)^{m} & \sim g_{\mathrm{s}}^{2 n} \ell_{\mathrm{s}}^{2 m} \bar{M}_{\mathrm{s}}^{-2 m / 7}\left(\frac{Q p_{0}}{M}\right)^{-2 m / 7}\left(w_{a b c d}\right)^{m}\left(1-\frac{64}{49 p_{0}^{2}}\right)^{3(m-7 n) / 14} \\
& \sim g_{\mathrm{s}}^{2 n-2 m} L^{16 m}\left(w_{a b c d}\right)^{m}\left(\frac{Q p_{0}}{M}\right)^{-2 m / 7}\left(1-\frac{64}{49 p_{0}^{2}}\right)^{3(m-7 n) / 14} \\
& \sim g_{\mathrm{s}}^{2 n-2 m} L^{16 m}
\end{aligned}
$$

where $L$ is defined by eq. (2.29). In the last line, we simply dropped $\frac{Q}{M}$ dependence, which deeply depends on the structure of each higher derivative term. Therefore we roughly estimate that the entropy of boosted quantum black string is valid when $L \leq 1$ and $1 \ll g_{\mathrm{s}}^{2}$, if each higher derivative term has a coefficient of order unity. This will also be true for the boosted quantum black hole.

\section{Near horizon limit of the boosted quantum black string and its insta- bility}

The gauge/gravity correspondence is a powerful tool to investigate the nonperturbative aspects of the gauge theory [40-42]. In this section we consider the near horizon limit of $N$ D0-branes, which corresponds to the infinitely boosted $(\beta \rightarrow \infty)$ quantum black string [43]. The momentum of the boost corresponds to the charge of $N$ D0-branes, and is expressed as

$$
Q_{\mathrm{s}}=\frac{N}{\ell_{\mathrm{s}} g_{\mathrm{s}}} \text {. }
$$

Since the boost parameter is given by $\alpha^{7}=1 / \sinh ^{2} \beta$, the near horizon limit corresponds to $\alpha \rightarrow 0$. While taking this limit, we should fix the 't Hooft coupling $\lambda$ and the energy scale $U_{0}$ of the gauge theory on $N$ D0-branes, which are written as

$$
\lambda=\frac{g_{\mathrm{s}} N}{(2 \pi)^{2} \ell_{\mathrm{s}}^{3}}, \quad U_{0}=\frac{r_{\mathrm{s}}}{\ell_{\mathrm{s}}^{2}} .
$$


From eqs. (3.10) and (4.1), $\alpha$ approaches to zero like

$$
\alpha^{7} \rightarrow \frac{7 V_{S^{8}} \ell_{\mathrm{S}}^{4} U_{0}^{7}}{(2 \pi)^{9} \lambda}
$$

and $\ell_{\mathrm{S}}$ also goes to zero in the limit.

Now we take the near horizon limit for the boosted quantum black string. First of all, $Q_{\mathrm{s}}$ is exactly given by eq. (4.1), so it is expressed in terms of $\ell_{\mathrm{s}}, \lambda$ and $N$ as

$$
Q_{\mathrm{s}}=\frac{N^{2}}{(2 \pi)^{2} \ell_{\mathrm{s}}^{4} \lambda}
$$

This approaches to the infinity by taking the near horizon limit. Next, $M_{\mathrm{S}}$ is given by eq. (3.10) and it behaves like

$$
\begin{aligned}
M_{\mathrm{s}} & =Q_{\mathrm{s}} \frac{8 \alpha^{7}}{7 \sqrt{1+\alpha^{7}}}\left(1+\frac{7}{8 \alpha^{7}}-\frac{2108160 \gamma}{r_{\mathrm{s}}^{6}}\right) \\
& \rightarrow \frac{N^{2}}{(2 \pi)^{2} \ell_{\mathrm{s}}^{4} \lambda}\left(1+\frac{3 \ell_{\mathrm{s}}^{4} U_{0}^{7}}{35(2 \pi)^{5} \lambda}-\frac{61 \pi \ell_{\mathrm{s}}^{4} U_{0} \lambda}{7 N^{2}}\right) .
\end{aligned}
$$

By taking the near horizon limit, the leading term diverges like the charge. However, the internal energy $E_{\mathrm{s}}=M_{\mathrm{s}}-Q_{\mathrm{s}}$ becomes finite and is given by

$$
\tilde{E}_{\mathrm{s}}=\frac{3 N^{2}}{(2 \pi)^{7} 35}\left(\tilde{U}_{0}^{7}-\frac{9760 \pi^{6} \tilde{U}_{0}}{3 N^{2}}\right)
$$

where $\tilde{E}_{\mathrm{s}} \equiv E_{\mathrm{s}} / \lambda^{1 / 3}$ and $\tilde{U}_{0} \equiv U_{0} / \lambda^{1 / 3}$. The temperature (3.9) approaches to

$$
\tilde{T}_{\mathrm{s}}=a_{1} \tilde{U}_{0}^{5 / 2}\left(1-\frac{2440 \pi^{6}}{7 N^{2} \tilde{U}_{0}^{6}}\right), \quad a_{1} \equiv \frac{7}{2^{4}\left(15 \pi^{7}\right)^{1 / 2}},
$$

where $\tilde{T}_{\mathrm{s}} \equiv T_{\mathrm{s}} / \lambda^{1 / 3}$. Inversely solving this, we obtain

$$
\tilde{U}_{0}=a_{1}^{-2 / 5} \tilde{T}_{\mathrm{s}}^{2 / 5}\left(1+\frac{976 \pi^{6} a_{1}^{12 / 5}}{7 N^{2} \tilde{T}_{\mathrm{s}}^{12 / 5}}\right) .
$$

Thus physical quantities can be expressed in terms of $\tilde{T}_{\mathrm{s}}$ and $N$. Finally, the near horizon limit of the entropy (3.13) is given by

$$
\begin{aligned}
S_{\mathrm{S}} & =\frac{N^{2} \tilde{U}_{0}^{9 / 2}}{28\left(15 \pi^{7}\right)^{1 / 2}}\left(1+\frac{2440 \pi^{6}}{7 N^{2} \tilde{U}_{0}^{6}}\right) \\
& =\frac{4 N^{2} \tilde{T}_{\mathrm{s}}^{9 / 5}}{49 a_{1}^{4 / 5}}\left(1+\frac{976 \pi^{6} a_{1}^{12 / 5}}{N^{2} \tilde{T}_{\mathrm{s}}^{12 / 5}}\right) \\
& \sim 11.5 N^{2} \tilde{T}_{\mathrm{s}}^{9 / 5}\left(1+\frac{0.334}{N^{2} \tilde{T}_{\mathrm{S}}^{12 / 5}}\right) .
\end{aligned}
$$


This expression is the generalization of the discussion in ref. [43], and first derived in ref. [34] by evaluating in the background of the near horizon geometry.

Let us examine the Gregory-Laflamme instability of the boosted quantum black string in the near horizon limit. In order to do this, we need to compare the entropy (4.9) with that of the boosted quantum black hole. By taking the near horizon limit of eq. (3.14), the entropy of the boosted quantum black hole is expressed as

$$
\begin{aligned}
S_{\mathrm{h}} & =\frac{N^{15 / 8} \tilde{U}_{0}^{63 / 16}}{3 \sqrt{2}(105)^{9 / 16} \pi^{47 / 16}}\left(1-\frac{1830 \pi^{6}}{N^{2} \tilde{U}_{0}^{6}}+\frac{10503(105)^{3 / 8} \pi^{21 / 8}}{2^{10} N^{5 / 4} \tilde{U}_{0}^{21 / 8}}\right) \\
& =\frac{N^{15 / 8} \tilde{T}_{\mathrm{s}}^{63 / 40}}{3 \sqrt{2}(105)^{9 / 16} \pi^{47 / 16} a_{1}^{63 / 40}}\left(1-\frac{1281 \pi^{6} a_{1}^{12 / 5}}{N^{2} \tilde{T}_{\mathrm{s}}^{12 / 5}}+\frac{10503(105)^{3 / 8} \pi^{21 / 8} a_{1}^{21 / 20}}{2^{10} N^{5 / 4} \tilde{T}_{\mathrm{s}}^{21 / 20}}\right) \\
& \sim 10.2 N^{15 / 8} \tilde{T}_{\mathrm{s}}^{63 / 40}\left(1-\frac{0.438}{N^{2} \tilde{T}_{\mathrm{s}}^{12 / 5}}+\frac{1.79}{N^{5 / 4} \tilde{T}_{\mathrm{s}}^{21 / 20}}\right)
\end{aligned}
$$

It is worth noting that the leading part behaves like $N^{15 / 8}$ and the quantum effects consist of two terms. It is challenging problem to explain these behaviors from the gauge theory on $N$ D0-branes.

The instability of the near horizon limit of the boosted quantum black string is investigated by comparing eq. (4.9) with eq. (4.10). Up to the next leading order, the ratio of the entropies is given by

$$
\begin{aligned}
\frac{S_{\mathrm{h}}}{S_{\mathrm{S}}} & \sim L\left(1-\frac{2257 \pi^{6} a_{1}^{12 / 5}}{N^{2} \tilde{T}_{\mathrm{s}}^{12 / 5}}+\frac{10503(105)^{3 / 8} \pi^{21 / 8} a_{1}^{21 / 20}}{2^{10} N^{5 / 4} \tilde{T}_{\mathrm{s}}^{21 / 20}}\right) \\
& \sim L\left(1-\frac{0.772}{N^{2} \tilde{T}_{\mathrm{S}}^{12 / 5}}+\frac{1.79}{N^{5 / 4} \tilde{T}_{\mathrm{s}}^{21 / 20}}\right), \\
& \sim L\left(1-2.93 \frac{L^{32 / 3}}{N^{2 / 3}}+3.21 \frac{L^{14 / 3}}{N^{2 / 3}}\right),
\end{aligned}
$$

where

$$
L \equiv \frac{49}{12 \sqrt{2}(105)^{9 / 16} \pi^{47 / 16} a_{1}^{31 / 40} N^{1 / 8} \tilde{T}_{\mathrm{S}}^{9 / 40}} \sim \frac{0.882}{N^{1 / 8} \tilde{T}_{\mathrm{S}}^{9 / 40}} .
$$

The transition of the boosted quantum black string in the near horizon limit occurs at

$$
L=1-\frac{0.277}{N^{2 / 3}}
$$

up to $\mathcal{O}\left(N^{-4 / 3}\right)$. This result is consistent with the discussion in ref. [43] in the classical limit.

Let us examine the validity of eq. (4.11) by estimating the other higher derivative terms. The near horizon limit of eq. (3.17) is given by

$$
\begin{aligned}
g_{\mathrm{s}} e^{\phi} & \sim \frac{\tilde{U}_{0}^{-21 / 4}}{N} \sim \frac{\tilde{T}_{\mathrm{s}}^{-21 / 10}}{N} \sim N^{1 / 6} L^{28 / 3}, \\
\ell_{\mathrm{s}}^{2} R_{a b c d} & \sim \tilde{U}_{0}^{3 / 2} \sim \tilde{T}_{\mathrm{s}}^{3 / 5} \sim N^{-1 / 3} L^{-8 / 3},
\end{aligned}
$$




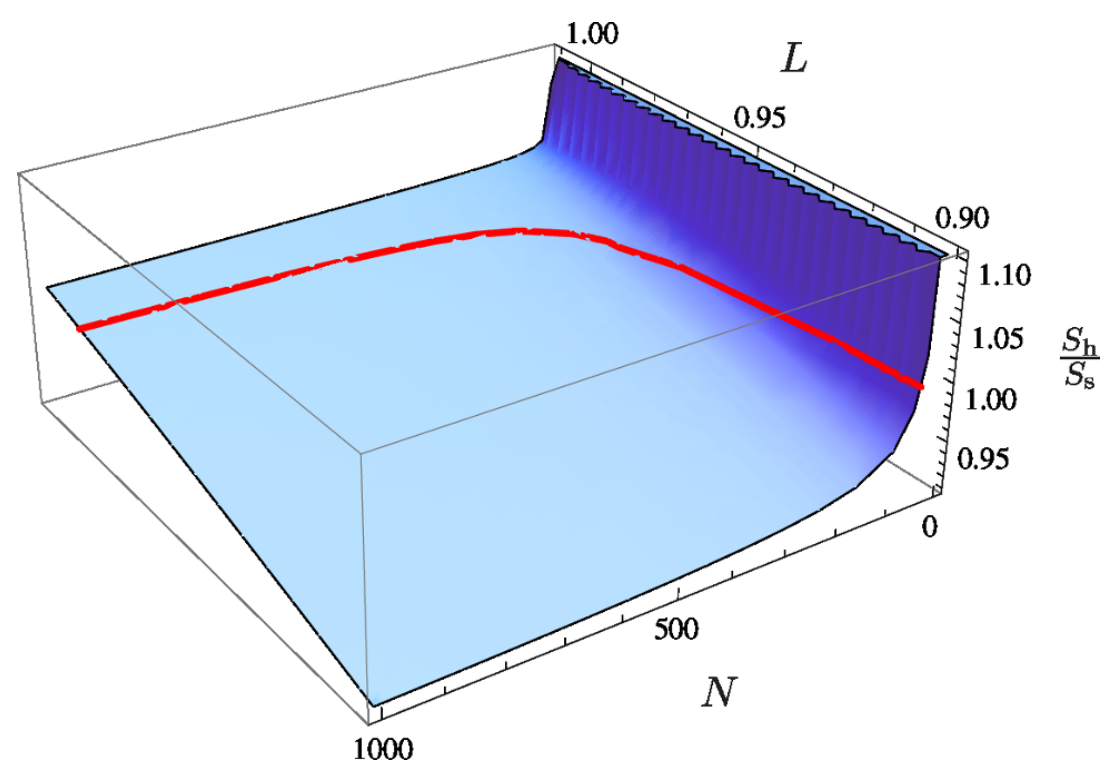

Figure 3. Plot of eq. (4.11) which is valid when $L \leq 1$ and $L^{-56} \ll N$. A red line correspond to $S_{\mathrm{h}}=S_{\mathrm{s}}$.

and a generic term is estimated as

$$
\left(g_{\mathrm{s}}^{2} e^{2 \phi}\right)^{n}\left(\ell_{\mathrm{S}}^{2} R_{a b c d}\right)^{m} \sim \frac{\tilde{T}_{\mathrm{s}}^{(3 m-21 n) / 5}}{N^{2 n}} \sim N^{(n-m) / 3} L^{8(7 n-m) / 3} .
$$

The leading tree, 1-loop and $n(\geq 2)$-loop corrections in the type IIA superstring theory become $(n, m)=(0,3),(1,3),(n, n+3)$, respectively. So the estimations of leading tree, 1-loop and $n(\geq 2)$-loop are given by $N^{-1} L^{-8}, N^{-2 / 3} L^{32 / 3}$ and $N^{-1} L^{16 n-8}$, respectively. Then the eq. (4.11) is valid when

$$
L \leq 1, \quad L^{-56} \ll N,
$$

if each higher derivative term has a coefficient of order unity. The plot of eq. (4.11) is drawn in figure 3.

\section{Conclusion and discussion}

In this paper we explored the quantum nature of the black hole and black string in 11 dimensions by taking into account the higher derivative $R^{4}$ corrections. Especially we clarified the transition from the quantum black string to the quantum black hole from entropic arguments.

First we constructed the solutions of quantum black hole and black string up to the linear order of $\ell_{\mathrm{p}}^{6}$. These are asymptotically flat, but the behaviors near the event horizons are quite different from the classical ones. We also investigated the thermodynamics of these quantum solutions, which satisfy the first law of the thermodynamics. The entropies of both quantum black hole and black string increase because of the quantum corrections. 
By comparing these two, we discussed the quantum nature of the Gregory-Laflamme instability. When the string coupling constant $g_{\mathrm{s}}$ is quite large, it is reasonable to trust the classical analyses and from eq. (2.30) the transition occurs around

$$
M \sim 0.412 g_{\mathrm{s}}^{5}+8.09 g_{\mathrm{s}} .
$$

On the other hand, when $g_{\mathrm{s}} \sim 1$, we should take into account other higher derivative corrections more seriously. Notice also that we neglected the effect of the circle compactification on the black hole to derive the above transition point. The effect of the compactification on the black hole is investigated in ref. [44], and corrections to the Gregory-Laflamme instability are explored analytically when the mass of the black hole is small in refs. [45, 46]. By consulting the result in ref. [46], we see that the entropy (2.26) is modified into

$$
\begin{aligned}
S_{\mathrm{h}} & =8 \pi^{2}\left(\frac{g_{\mathrm{s}}^{3} M^{9}}{9^{9} V_{S^{9}}}\right)^{1 / 8}\left\{1+\frac{10503}{2^{11} \pi^{4}}\left(\frac{9^{8} V_{S^{9}}}{8^{8} V_{S^{8}}}\right)^{6} \frac{L^{42}}{g_{\mathrm{s}}^{4}}+\frac{\zeta(8)}{16 V_{S^{9}}} \frac{\left(8^{8} V_{S^{8}}\right)^{8}}{\left(9^{9} V_{S^{9}}\right)^{7}} \frac{1}{L^{56}}\right\} \\
& \sim 8 \pi^{2}\left(\frac{g_{\mathrm{s}}^{3} M^{9}}{9^{9} V_{S^{9}}}\right)^{1 / 8}\left(1+6.04 \frac{L^{42}}{g_{\mathrm{s}}^{4}}+0.00101 \frac{1}{L^{56}}\right) .
\end{aligned}
$$

The third term in the parenthesis corresponds to the effect of the circle compactification. Therefore when the parameters are in the region of $L \sim 1$ and $1<g_{\mathrm{s}}<5$, the effect of the compactification is negligible compared to the quantum correction. And when the parameters are in the region of $L \sim 1$ and $5 \leq g_{\mathrm{s}}$, both effects are negligible compared to the leading part. When the mass of the black hole is not small, we have to employ numerical calculation [47].

Second we boosted the quantum black hole and black string solutions, and showed that the latter corresponds to the nonextremal quantum black 0-brane solution. Both entropies depend on the boost parameter in a complicated way, and because of this, the transition occurs for larger $M$ than eq. (5.1). It is interesting to note that there appear another transition point around $g_{\mathrm{s}} \sim 4$ for $\frac{Q}{M}=0.9$.

Finally we consider the near horizon limit of the boosted quantum black string. In this limit, the boost parameter goes to the infinity, and physical quantities are expressed in terms of the temperature. From eq. (4.13) the transition occurs around

$$
T_{\mathrm{s}} \sim \frac{0.574}{N^{5 / 9}}+\frac{0.707}{N^{11 / 9}} .
$$

This shows that quantum effects become important when the number of D0-branes $N$ becomes small.

As a future work it is interesting to understand the Gregory-Laflamme instability in terms of the dual gauge theory. In fact numerical study of the thermal D0-branes system has been investigated considerably in refs. [48]-[52], and especially the corresponding instability is numerically discussed in ref. [51]. It is also of great interest to understand the GregoryLaflamme instability of the black string from the gauge theory side which is not stretching to the 11 th direction $[53,54]$. In this paper we focused on $g_{\mathrm{s}}$ correction in the type IIA superstring theory, but it seems to be possible to examine $\alpha^{\prime}$ correction as well. Finally the confirmations of the relation between thermodynamic and perturbative instabilities are important directions [5, 55-57]. 


\section{Acknowledgments}

The author would like to thank Takanori Fujiwara, Yoshinobu Habara, Yosuke Imamura, Katsushi Ito, Tetsuji Kimura, Yusuke Kimura, Tatsuma Nishioka, Makoto Sakaguchi, Hidehiko Shimada, Fumihiko Sugino and Shinya Tomizawa. This work was partially supported by the Ministry of Education, Science, Sports and Culture, Grant-in-Aid for Young Scientists (B) 24740140, 2012.

\section{A Calculations on quantum black hole}

\section{A.1 Explicit values of tensors}

First we choose the vielbein of the quantum black hole as follows.

$$
\begin{aligned}
& e^{0}=r_{\mathrm{h}} B_{1}^{-1 / 2} A_{1}^{1 / 2} d \tau, \quad e^{1}=r_{\mathrm{h}} A_{1}^{-1 / 2} d x, \quad e^{2}=r_{\mathrm{h}} x d \theta_{1}, \\
& e^{3}=r_{\mathrm{h}} x \cos \theta_{1} d \theta_{2}, \quad e^{4}=r_{\mathrm{h}} x \cos \theta_{1} \cos \theta_{2} d \theta_{3}, \quad e^{5}=r_{\mathrm{h}} x \cos \theta_{1} \cos \theta_{2} \sin \theta_{3} d \theta_{4}, \\
& e^{6}=r_{\mathrm{h}} x \cos \theta_{1} \sin \theta_{2} d \theta_{5}, \quad e^{7}=r_{\mathrm{h}} x \cos \theta_{1} \sin \theta_{2} \sin \theta_{5} d \theta_{6}, \quad e^{8}=r_{\mathrm{h}} x \sin \theta_{1} d \theta_{7}, \\
& e^{9}=r_{\mathrm{h}} x \sin \theta_{1} \sin \theta_{7} d \theta_{8}, \quad e^{10}=r_{\mathrm{h}} x \sin \theta_{1} \cos \theta_{7} d \theta_{9},
\end{aligned}
$$

where $A_{1}(x)=1-\frac{1}{x^{8}}+\frac{\gamma}{r_{\mathrm{h}}^{6}} a_{1}(x)$ and $B_{1}(x)=1+\frac{\gamma}{r_{\mathrm{h}}^{6}} b_{1}(x)$. Then, up to the linear order of $\gamma$, nonzero components of the Riemann tensor, Ricci tensor and scalar curvature are calculated as

$$
\begin{aligned}
R_{0101} & =-\frac{36}{r_{\mathrm{h}}^{2} x^{10}}+\gamma \frac{x^{9} a_{1}^{\prime \prime}+x\left(1-x^{8}\right) b_{1}^{\prime \prime}-12 b_{1}^{\prime}}{2 r_{\mathrm{h}}^{8} x^{9}}, \\
R_{0 \hat{i} \hat{0} \hat{i}} & =\frac{4}{r_{\mathrm{h}}^{2} x^{10}}+\gamma \frac{x^{8} a_{1}^{\prime}+\left(1-x^{8}\right) b_{1}^{\prime}}{2 r_{\mathrm{h}}^{8} x^{9}}, \\
R_{1 \hat{i} 1 \hat{i}} & =-\frac{4}{r_{\mathrm{h}}^{2} x^{10}}-\gamma \frac{a_{1}^{\prime}}{2 r_{\mathrm{h}}^{8} x}, \quad R_{\hat{i} \hat{j} \hat{i} \hat{j}}=\frac{1}{r_{\mathrm{h}}^{2} x^{10}}-\gamma \frac{a_{1}}{r_{\mathrm{h}}^{8} x^{2}}, \\
R_{00} & =\gamma \frac{x^{9} a_{1}^{\prime \prime}+9 x^{8} a_{1}^{\prime}+x\left(1-x^{8}\right) b_{1}^{\prime \prime}-3\left(1+3 x^{8}\right) b_{1}^{\prime}}{2 r_{\mathrm{h}}^{8} x^{9}}, \\
R_{11} & =\gamma \frac{-x^{9} a_{1}^{\prime \prime}-9 x^{8} a_{1}^{\prime}-x\left(1-x^{8}\right) b_{1}^{\prime \prime}+12 b_{1}^{\prime}}{2 r_{\mathrm{h}}^{8} x^{9}}, \\
R_{\hat{i} \hat{i}} & =\gamma \frac{-2 x^{8} a_{1}^{\prime}-16 x^{7} a_{1}-\left(1-x^{8}\right) b_{1}^{\prime}}{2 r_{\mathrm{h}}^{8} x^{9}}, \\
R & =\gamma \frac{-x^{9} a_{1}^{\prime \prime}-18 x^{8} a_{1}^{\prime}-72 x^{7} a_{1}-x\left(1-x^{8}\right) b_{1}^{\prime \prime}+3\left(1+3 x^{8}\right) b_{1}^{\prime}}{r_{\mathrm{h}}^{8} x^{9}},
\end{aligned}
$$

where $\hat{i}, \hat{j}=2, \cdots, 10$ and $\hat{i} \neq \hat{j}$. 
Next we evaluate higher derivative terms up to $\mathcal{O}(\gamma)$. Nonzero components of $X_{a b c d}$, $R X_{i j} \equiv R_{a b c i} X^{a b c}{ }_{j}$ and $D D X_{i j} \equiv D_{(a} D_{b)} X^{a}{ }_{i j}{ }^{b}$ are evaluated as

$$
\begin{aligned}
X_{0101} & =-\frac{44070912}{r_{\mathrm{h}}^{6} x^{30}}, \quad X_{0 \hat{i} \hat{0} \hat{i}}=-X_{1 \hat{i} \hat{i} \hat{i}}=-\frac{2844672}{r_{\mathrm{h}}^{6} x^{30}}, \quad X_{\hat{i} \hat{j} \hat{i} \hat{j}}=\frac{1949952}{r_{\mathrm{h}}^{6} x^{30}}, \\
R X_{00} & =-R X_{11}=-\frac{2968289280}{r_{\mathrm{h}}^{8} x^{40}}, \quad R X_{\hat{i} \hat{i}}=-\frac{14315520}{r_{\mathrm{h}}^{8} x^{40}}, \\
D D X_{00} & =-\frac{1902182400\left(13-11 x^{8}\right)}{r_{\mathrm{h}}^{8} x^{40}}, \quad D D X_{11}=\frac{900\left(3445248+781824 x^{8}\right)}{r_{\mathrm{h}}^{8} x^{40}}, \\
D D X_{\hat{i} \hat{i}} & =\frac{78182400\left(31-23 x^{8}\right)}{r_{\mathrm{h}}^{8} x^{40}}, \quad t_{8} t_{8} R^{4}-\frac{1}{4 !} \epsilon_{11} \epsilon_{11} R^{4}=\frac{1451934720}{r_{\mathrm{h}}^{8} x^{40}},
\end{aligned}
$$

where $\hat{i}, \hat{j}=2, \cdots, 10$ and $\hat{i} \neq \hat{j}$.

\section{A.2 Plots of $A_{1}(x)$}

Let us examine the properties of $A_{1}(x)$ given in section 2.2. For simplicity we just set $c_{\mathrm{h}}=0$ below, so $A_{1}(x)$ is given by

$$
A_{1}(x)=1-\frac{1}{x^{8}}+\tilde{\gamma}\left(\frac{422707200}{x^{30}}-\frac{349736448}{x^{38}}\right), \quad \tilde{\gamma}=\frac{\gamma}{r_{\mathrm{h}}^{6}} .
$$

The derivative of $A_{1}(x)$ is calculated as

$$
A_{1}^{\prime}(x)=\frac{8 \tilde{\gamma}}{x^{39}}\left(\tilde{\gamma}^{-1} x^{30}-1585152000 x^{8}+1661248128\right) .
$$

Then $A_{1}^{\prime}(x)=0$ has one or two solutions when the minimum of the function in the parentheses becomes zero or negative, respectively. The function in the parentheses becomes minimum when $x^{22}=422707200 \tilde{\gamma}$, and the minimum takes negative value when

$$
-1162444800(422707200 \tilde{\gamma})^{4 / 11}+1661248128<0 \Leftrightarrow 6.32 \times 10^{-9}<\tilde{\gamma} .
$$

Plots of $A_{1}(x)$ with $\tilde{\gamma}=10^{-9}$ and $10^{-8}$ are shown in figure 4 . In both cases, locations of the event horizons are shifted inward compared with the classical case. Especially the behavior of $A_{1}(x)$ with $\tilde{\gamma}=10^{-8}$ is quite different around the event horizon, so a test particle feels a repulsive force.

\section{B Calculations on quantum black string}

\section{B.1 Explicit values of tensors}

First we choose the vielbein of the quantum black hole as follows.

$$
\begin{aligned}
& e^{0}=r_{\mathrm{s}} G_{1}^{-1 / 2} F_{1}^{1 / 2} d \tau, \quad e^{1}=r_{\mathrm{s}} F_{1}^{-1 / 2} d x, \quad e^{2}=r_{\mathrm{s}} x d \theta_{1}, \\
& e^{3}=r_{\mathrm{s}} x \cos \theta_{1} d \theta_{2}, \quad e^{4}=r_{\mathrm{s}} x \cos \theta_{1} \cos \theta_{2} d \theta_{3}, \quad e^{5}=r_{\mathrm{s}} x \cos \theta_{1} \cos \theta_{2} \sin \theta_{3} d \theta_{4}, \\
& e^{6}=r_{\mathrm{s}} x \cos \theta_{1} \sin \theta_{2} d \theta_{5}, \quad e^{7}=r_{\mathrm{s}} x \cos \theta_{1} \sin \theta_{2} \sin \theta_{5} d \theta_{6}, \quad e^{8}=r_{\mathrm{s}} x \sin \theta_{1} d \theta_{7}, \\
& e^{9}=r_{\mathrm{s}} x \sin \theta_{1} \sin \theta_{7} d \theta_{8}, \quad e^{\natural}=r_{\mathrm{s}} G_{2}^{1 / 2} d y,
\end{aligned}
$$




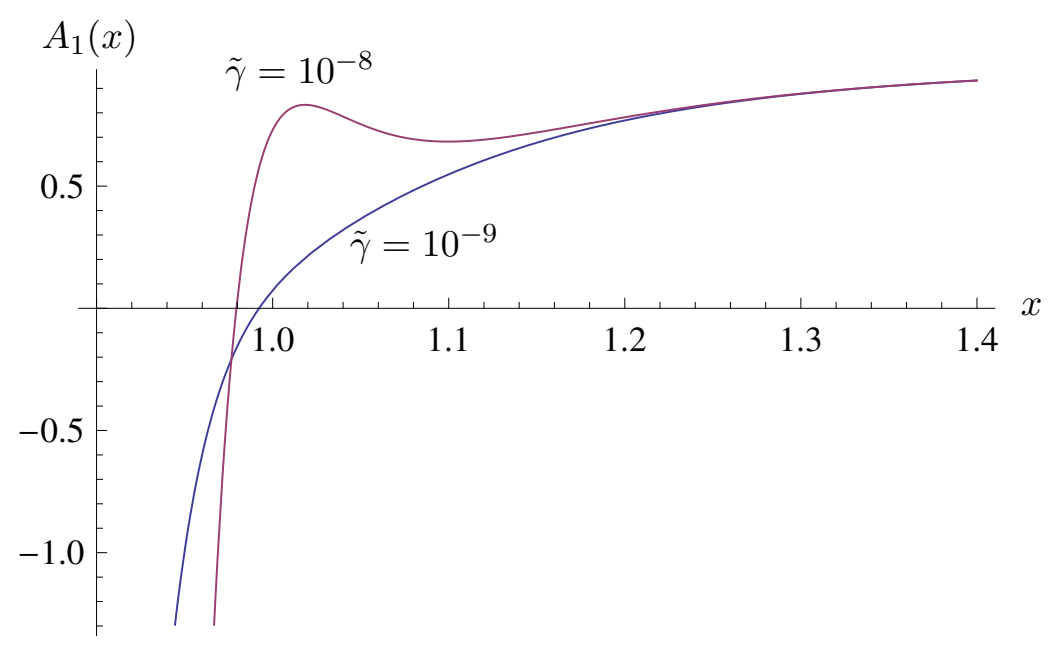

Figure 4. Plots of $A_{1}(x)$ with $\tilde{\gamma}=10^{-9}$ and $10^{-8}$.

where $F_{1}(x)=1-\frac{1}{x^{7}}+\frac{\gamma}{r_{\mathrm{s}}^{6}} f_{1}(x)$ and $G_{i}(x)=1+\frac{\gamma}{r_{\mathrm{s}}^{6}} g_{i}(x)$. Then, up to the linear order of $\gamma$, nonzero components of the Riemann tensor, Ricci tensor and scalar curvature are calculated as

$$
\begin{aligned}
& R_{0101}=-\frac{28}{r_{\mathrm{s}}^{2} x^{9}}+\gamma \frac{2 x^{8} f_{1}^{\prime \prime}+2 x\left(1-x^{7}\right) g_{1}^{\prime \prime}-21 g_{1}^{\prime}}{4 r_{\mathrm{s}}^{8} x^{8}}, \quad R_{0 \hat{i} 0 \hat{i}}=\frac{7}{2 r_{\mathrm{s}}^{2} x^{9}}+\gamma \frac{x^{7} f_{1}^{\prime}+\left(1-x^{7}\right) g_{1}^{\prime}}{2 r_{\mathrm{s}}^{8} x^{8}}, \\
& R_{0 \text { ฤด }}=\gamma \frac{7 g_{2}^{\prime}}{4 r_{\mathrm{s}}^{8} x^{8}}, \quad R_{1 \hat{i} 1 \hat{i}}=-\frac{7}{2 r_{\mathrm{s}}^{2} x^{9}}-\gamma \frac{f_{1}^{\prime}}{2 r_{\mathrm{s}}^{8} x}, \quad R_{1 \natural 1 \natural}=\gamma \frac{2 x\left(1-x^{7}\right) g_{2}^{\prime \prime}-7 g_{2}^{\prime}}{4 r_{\mathrm{s}}^{8} x^{8}}, \\
& R_{\hat{\hat{j}} \hat{i} \hat{i} \hat{j}}=\frac{1}{r_{\mathrm{s}}^{2} x^{9}}-\gamma \frac{f_{1}}{r_{\mathrm{s}}^{8} x^{2}}, \quad R_{\hat{i} \downarrow \hat{\imath} \mathfrak{}}=\gamma \frac{\left(1-x^{7}\right) g_{2}^{\prime}}{2 r_{\mathrm{s}}^{8} x^{8}}, \\
& R_{00}=\gamma \frac{2 x^{8} f_{1}^{\prime \prime}+16 x^{7} f_{1}^{\prime}+2 x\left(1-x^{7}\right) g_{1}^{\prime \prime}-\left(5+16 x^{7}\right) g_{1}^{\prime}+7 g_{2}^{\prime}}{4 r_{\mathrm{s}}^{8} x^{8}}, \\
& R_{11}=\gamma \frac{-2 x^{8} f_{1}^{\prime \prime}-16 x^{7} f_{1}^{\prime}-2 x\left(1-x^{7}\right) g_{1}^{\prime \prime}+21 g_{1}^{\prime}+2 x\left(1-x^{7}\right) g_{2}^{\prime \prime}-7 g_{2}^{\prime}}{4 r_{\mathrm{s}}^{8} x^{8}}, \\
& R_{\hat{i} \hat{i}}=\gamma \frac{-2 x^{7} f_{1}^{\prime}-14 x^{6} f_{1}-\left(1-x^{7}\right) g_{1}^{\prime}+\left(1-x^{7}\right) g_{2}^{\prime}}{2 r_{\mathrm{s}}^{8} x^{8}}, \quad R_{\text {孔中 }}=\gamma \frac{x\left(1-x^{7}\right) g_{2}^{\prime \prime}+\left(1-8 x^{7}\right) g_{2}^{\prime}}{2 r_{\mathrm{s}}^{8} x^{8}}, \\
& R=\gamma \frac{-2 x^{8} f_{1}^{\prime \prime}-32 x^{7} f_{1}^{\prime}-112 x^{6} f_{1}-2 x\left(1-x^{7}\right) g_{1}^{\prime \prime}+\left(5+16 x^{7}\right) g_{1}^{\prime}+2 x\left(1-x^{7}\right) g_{2}^{\prime \prime}+2\left(1-8 x^{7}\right) g_{2}^{\prime}}{2 r_{\mathrm{s}}^{8} x^{8}},
\end{aligned}
$$

where $\hat{i}, \hat{j}=2, \cdots, 9$ and $\hat{i} \neq \hat{j}$.

Next we evaluate higher derivative terms up to $\mathcal{O}(\gamma)$. Nonzero components of $X_{a b c d}$, $R X_{i j} \equiv R_{a b c i} X^{a b c}{ }_{j}$ and $D D X_{i j} \equiv D_{(a} D_{b)} X^{a}{ }_{i j}{ }^{b}$ are evaluated as

$$
\begin{aligned}
& X_{0101}=-\frac{20321280}{r_{\mathrm{s}}^{6} x^{27}}, \quad X_{0 \hat{i} 0 \hat{i}}=-X_{1 \hat{i} 1 \hat{i}}=-\frac{1270080}{r_{\mathrm{s}}^{6} x^{27}}, \quad X_{\hat{i} \hat{j} \hat{i} \hat{j}}=\frac{1192320}{r_{\mathrm{s}}^{6} x^{27}}, \\
& R X_{00}=-R X_{11}=-\frac{1066867200}{r_{\mathrm{s}}^{8} x^{36}}, \quad R X_{\hat{i} \hat{i}}=-\frac{1088640}{r_{\mathrm{s}}^{8} x^{36}},
\end{aligned}
$$




$$
\begin{aligned}
& D D X_{00}=\frac{198132480\left(-47+40 x^{7}\right)}{r_{\mathrm{s}}^{8} x^{36}}, \quad D D X_{11}=\frac{1701\left(1313280+317440 x^{7}\right)}{2 r_{\mathrm{s}}^{8} x^{36}}, \\
& D D X_{\hat{i} \hat{i}}=\frac{236234880\left(4-3 x^{7}\right)}{r_{\mathrm{s}}^{8} x^{36}}, \quad t_{8} t_{8} R^{4}-\frac{1}{4 !} \epsilon_{11} \epsilon_{11} R^{4}=\frac{531256320}{r_{\mathrm{s}}^{8} x^{36}},
\end{aligned}
$$

where $\hat{i}, \hat{j}=2, \cdots, 9$ and $\hat{i} \neq \hat{j}$.

\section{B.2 Solution of eq. (2.19)}

Let us solve the equations of motion (2.19) for the quantum black string. First we consider the following combinations.

$$
\begin{aligned}
\frac{E_{1}+E_{2}}{16 x^{28}\left(1-x^{7}\right)}= & g_{1}^{\prime}+\frac{1}{8} x g_{2}^{\prime \prime}+\frac{4097640960}{x^{28}}=0 \\
\frac{E_{1}+E_{2}+8\left(E_{3}-E_{4}\right)}{2 x^{28}}= & -16 x^{7} f_{1}^{\prime}-112 x^{6} f_{1}-7 x\left(1-x^{7}\right) g_{2}^{\prime \prime}+56 x^{7} g_{2}^{\prime} \\
& +\frac{2525644800}{x^{28}}-\frac{10102579200}{x^{21}}=0 .
\end{aligned}
$$

These are solved as

$$
\begin{aligned}
& g_{1}=c_{1}-\frac{1}{8} x g_{2}^{\prime}+\frac{1}{8} g_{2}+\frac{151764480}{x^{27}} \\
& f_{1}=\frac{c_{2}}{x^{7}}-\frac{7\left(1-x^{7}\right)}{16 x^{6}} g_{2}^{\prime}+\frac{7}{16 x^{7}} g_{2}-\frac{5846400}{x^{34}}+\frac{31570560}{x^{27}} .
\end{aligned}
$$

By inserting these solutions into $E_{1}=0$, we obtain

$$
\begin{aligned}
\frac{E_{1}}{9 x^{28}} & =x\left(1-x^{7}\right) g_{2}^{\prime \prime}+\left(1-8 x^{7}\right) g_{2}^{\prime}+\frac{7640801280}{x^{28}}-\frac{5922201600}{x^{21}} \\
& =\left\{x\left(1-x^{7}\right) g_{2}^{\prime}-\frac{282992640}{x^{27}}+\frac{296110080}{x^{20}}\right\}^{\prime}=0 .
\end{aligned}
$$

From this $g_{2}(x)$ is solved as

$$
g_{2}(x)=c_{3}+c_{4} \log \frac{x^{7}}{x^{7}-1}-\frac{94330880}{9 x^{27}}+\frac{655872}{x^{20}}+\frac{13117440}{13 x^{13}}+\frac{2186240}{x^{6}}+1873920 I(x),
$$

where

$$
\begin{aligned}
I(x)= & \log \frac{x^{7}(x-1)}{x^{7}-1}-\sum_{n=1,3,5} \cos \frac{n \pi}{7} \log \left(x^{2}+2 x \cos \frac{n \pi}{7}+1\right) \\
& -2 \sum_{n=1,3,5} \sin \frac{n \pi}{7}\left\{\tan ^{-1}\left(\frac{x+\cos \frac{n \pi}{7}}{\sin \frac{n \pi}{7}}\right)-\frac{\pi}{2}\right\} \\
I^{\prime}(x)= & \frac{7(x-1)}{x\left(x^{7}-1\right)} .
\end{aligned}
$$

$c_{3}$ and $c_{4}$ are integral constants but should be zero so that the solution becomes asymptotically flat and $g_{2}(1)$ is finite. $g_{1}(x)$ and $f_{1}(x)$ are determined by using eq. (B.5). $c_{1}$ should be zero because of the asymptotic flatness but $c_{2}$ remains as a constant parameter. We have solved three out of four equations in (2.19), but the remaining equation is automatically satisfied. 


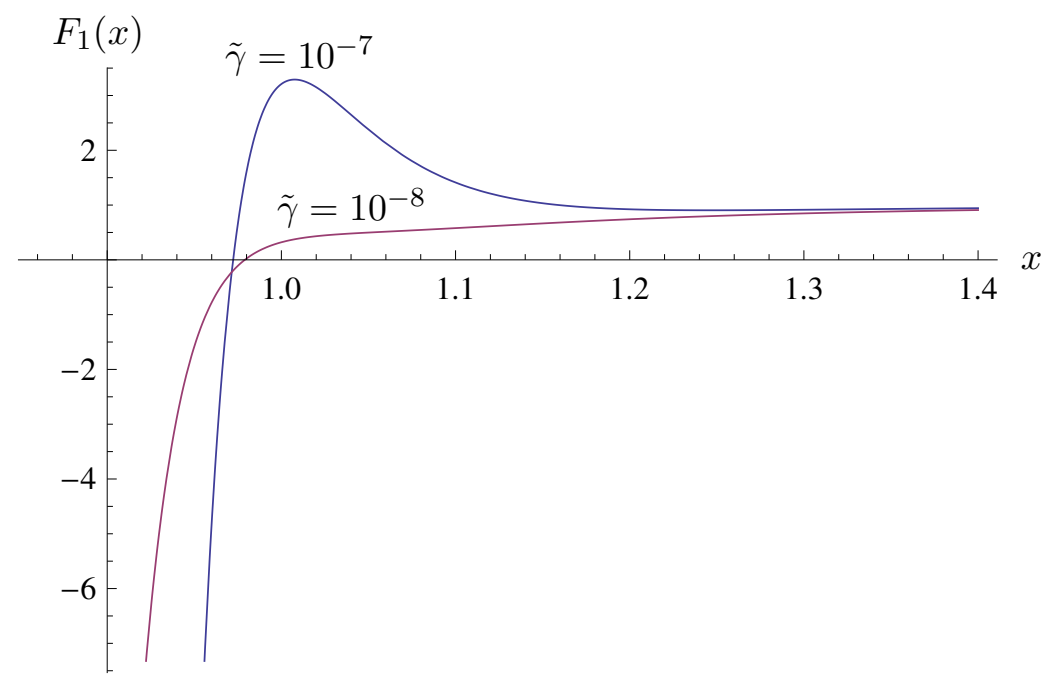

Figure 5. Plots of $F_{1}(x)$ with $\tilde{\gamma}=10^{-8}$ and $10^{-7}$.

\section{B.3 Plots of $F_{1}(x)$}

Let us examine the properties of $F_{1}(x)$ given in section 2.3. By taking into account the near horizon limit, we set $c_{\mathrm{s}}=3747840$ below, so $F_{1}(x)$ is given by

$$
\begin{aligned}
F_{1}(x)= & 1-\frac{1}{x^{7}}+\tilde{\gamma}\left(-\frac{1208170880}{9 x^{34}}+\frac{161405664}{x^{27}}+\frac{5738880}{13 x^{20}}\right. \\
& \left.+\frac{956480}{x^{13}}+\frac{3747840}{x^{7}}+\frac{819840}{x^{7}} I(x)\right), \quad \tilde{\gamma}=\frac{\gamma}{r_{\mathrm{h}}^{6}} .
\end{aligned}
$$

Plots of $F_{1}(x)$ with $\tilde{\gamma}=10^{-8}$ and $10^{-7}$ are shown in figure 5 . In both cases, locations of the event horizons are shifted inward compared with the classical case. Especially the behavior of $F_{1}(x)$ with $\tilde{\gamma}=10^{-7}$ is quite different around the event horizon, so a test particle feels a repulsive force [34].

Open Access. This article is distributed under the terms of the Creative Commons Attribution License (CC-BY 4.0), which permits any use, distribution and reproduction in any medium, provided the original author(s) and source are credited.

\section{References}

[1] P.K. Townsend, The eleven-dimensional supermembrane revisited, Phys. Lett. B 350 (1995) 184 [hep-th/9501068] [INSPIRE].

[2] E. Witten, String theory dynamics in various dimensions, Nucl. Phys. B 443 (1995) 85 [hep-th/9503124] [INSPIRE].

[3] E. Cremmer, B. Julia and J. Scherk, Supergravity Theory in Eleven-Dimensions, Phys. Lett. B 76 (1978) 409 [INSPIRE].

[4] M. Huq and M.A. Namazie, Kaluza-Klein Supergravity in Ten-dimensions, Class. Quant. Grav. 2 (1985) 293 [Erratum ibid. 2 (1985) 597] [INSPIRE]. 
[5] R. Gregory and R. Laflamme, Black strings and p-branes are unstable, Phys. Rev. Lett. 70 (1993) 2837 [hep-th/9301052] [INSPIRE].

[6] R. Gregory and R. Laflamme, The Instability of charged black strings and p-branes, Nucl. Phys. B 428 (1994) 399 [hep-th/9404071] [INSPIRE].

[7] G.T. Horowitz and K. Maeda, Fate of the black string instability, Phys. Rev. Lett. 87 (2001) 131301 [hep-th/0105111] [inSPIRE].

[8] S.S. Gubser, On nonuniform black branes, Class. Quant. Grav. 19 (2002) 4825 [hep-th/0110193] [INSPIRE].

[9] T. Wiseman, Static axisymmetric vacuum solutions and nonuniform black strings, Class. Quant. Grav. 20 (2003) 1137 [hep-th/0209051] [INSPIRE].

[10] H. Kudoh and T. Wiseman, Properties of Kaluza-Klein black holes, Prog. Theor. Phys. 111 (2004) 475 [hep-th/0310104] [INSPIRE].

[11] B. Kol, The Phase transition between caged black holes and black strings: A Review, Phys. Rept. 422 (2006) 119 [hep-th/0411240] [INSPIRE].

[12] D. Marolf, On the fate of black string instabilities: An Observation, Phys. Rev. D 71 (2005) 127504 [hep-th/0504045] [INSPIRE].

[13] B. Kleihaus, J. Kunz and E. Radu, New nonuniform black string solutions, JHEP 06 (2006) 016 [hep-th/0603119] [INSPIRE].

[14] L. Lehner and F. Pretorius, Black Strings, Low Viscosity Fluids and Violation of Cosmic Censorship, Phys. Rev. Lett. 105 (2010) 101102 [arXiv:1006.5960] [INSPIRE].

[15] E. Sorkin, A Critical dimension in the black string phase transition, Phys. Rev. Lett. 93 (2004) 031601 [hep-th/0402216] [INSPIRE].

[16] J.L. Hovdebo and R.C. Myers, Black rings, boosted strings and Gregory-Laflamme, Phys. Rev. D 73 (2006) 084013 [hep-th/0601079] [INSPIRE].

[17] G.T. Horowitz, Black holes in higher dimensions, Cambridge University Press (2012).

[18] D.J. Gross and E. Witten, Superstring Modifications of Einstein's Equations, Nucl. Phys. B 277 (1986) 1 [INSPIRE].

[19] D.J. Gross and J.H. Sloan, The Quartic Effective Action for the Heterotic String, Nucl. Phys. B 291 (1987) 41 [INSPIRE].

[20] M.T. Grisaru, A.E.M. van de Ven and D. Zanon, Four Loop $\beta$-function for the $N=1$ and $N=2$ Supersymmetric Nonlinear $\sigma$-model in Two-Dimensions, Phys. Lett. B 173 (1986) 423 [INSPIRE].

[21] M.T. Grisaru and D. Zanon, $\sigma$ Model Superstring Corrections to the Einstein-Hilbert Action, Phys. Lett. B 177 (1986) 347 [INSPIRE].

[22] A.A. Tseytlin, $R^{4}$ terms in 11 dimensions and conformal anomaly of $(2,0)$ theory, Nucl. Phys. B 584 (2000) 233 [hep-th/0005072] [INSPIRE].

[23] K. Becker and M. Becker, Supersymmetry breaking, M-theory and fluxes, JHEP 07 (2001) 038 [hep-th/0107044] [INSPIRE].

[24] M. de Roo, H. Suelmann and A. Wiedemann, Supersymmetric $R^{4}$ actions in ten-dimensions, Phys. Lett. B 280 (1992) 39 [InSPIRE]. 
[25] M. de Roo, H. Suelmann and A. Wiedemann, The Supersymmetric effective action of the heterotic string in ten-dimensions, Nucl. Phys. B 405 (1993) 326 [hep-th/9210099] [INSPIRE].

[26] H. Suelmann, Supersymmetry and string effective actions, Ph.D. Thesis, Groningen University, Groningen The Netherlands (1994).

[27] K. Peeters, P. Vanhove and A. Westerberg, Supersymmetric higher derivative actions in ten-dimensions and eleven-dimensions, the associated superalgebras and their formulation in superspace, Class. Quant. Grav. 18 (2001) 843 [hep-th/0010167] [INSPIRE].

[28] Y. Hyakutake and S. Ogushi, $R^{4}$ corrections to eleven dimensional supergravity via supersymmetry, Phys. Rev. D 74 (2006) 025022 [hep-th/0508204] [INSPIRE].

[29] Y. Hyakutake and S. Ogushi, Higher derivative corrections to eleven dimensional supergravity via local supersymmetry, JHEP 02 (2006) 068 [hep-th/0601092] [INSPIRE].

[30] Y. Hyakutake, Toward the Determination of $R^{3} F^{2}$ Terms in M-theory, Prog. Theor. Phys. 118 (2007) 109 [hep-th/0703154] [inSPIRE].

[31] C.G. Callan Jr., R.C. Myers and M.J. Perry, Black Holes in String Theory, Nucl. Phys. B 311 (1989) 673 [INSPIRE].

[32] Y. Brihaye, T. Delsate and E. Radu, Einstein-Gauss-Bonnet black strings, JHEP 07 (2010) 022 [arXiv: 1004.2164] [INSPIRE].

[33] A. Giacomini, J. Oliva and A. Vera, Black Strings in Gauss-Bonnet Theory are Unstable, Phys. Rev. D 91 (2015) 104033 [arXiv:1503.03696] [INSPIRE].

[34] Y. Hyakutake, Quantum near-horizon geometry of a black 0-brane, Prog. Theor. Exp. Phys. 2014 (2014) 033B04 [arXiv: 1311.7526] [INSPIRE].

[35] Y. Hyakutake, Quantum M-wave and Black 0-brane, JHEP 09 (2014) 075 [arXiv: 1407.6023] [INSPIRE].

[36] R.M. Wald, Black hole entropy is the Noether charge, Phys. Rev. D 48 (1993) 3427 [gr-qc/9307038] [INSPIRE].

[37] V. Iyer and R.M. Wald, Some properties of Noether charge and a proposal for dynamical black hole entropy, Phys. Rev. D 50 (1994) 846 [gr-qc/9403028] [InSPIRE].

[38] M.B. Green, J.G. Russo and P. Vanhove, Non-renormalisation conditions in type-II string theory and maximal supergravity, JHEP 02 (2007) 099 [hep-th/0610299] [INSPIRE].

[39] G.T. Horowitz and E.J. Martinec, Comments on black holes in matrix theory, Phys. Rev. D 57 (1998) 4935 [hep-th/9710217] [INSPIRE].

[40] J.M. Maldacena, The Large- $N$ limit of superconformal field theories and supergravity, Int. J. Theor. Phys. 38 (1999) 1113 [Adv. Theor. Math. Phys. 2 (1998) 231] [hep-th/9711200] [INSPIRE].

[41] S.S. Gubser, I.R. Klebanov and A.M. Polyakov, Gauge theory correlators from noncritical string theory, Phys. Lett. B 428 (1998) 105 [hep-th/9802109] [INSPIRE].

[42] E. Witten, Anti-de Sitter space and holography, Adv. Theor. Math. Phys. 2 (1998) 253 [hep-th/9802150] [INSPIRE].

[43] N. Itzhaki, J.M. Maldacena, J. Sonnenschein and S. Yankielowicz, Supergravity and the large- $N$ limit of theories with sixteen supercharges, Phys. Rev. D 58 (1998) 046004 [hep-th/9802042] [INSPIRE]. 
[44] R.C. Myers, Higher Dimensional Black Holes in Compactified Space-times, Phys. Rev. D 35 (1987) 455 [INSPIRE].

[45] B. Kol, E. Sorkin and T. Piran, Caged black holes: Black holes in compactified space-times. 1. Theory, Phys. Rev. D 69 (2004) 064031 [hep-th/0309190] [InSPIRE].

[46] T. Harmark, Small black holes on cylinders, Phys. Rev. D 69 (2004) 104015 [hep-th/0310259] [INSPIRE].

[47] E. Sorkin, B. Kol and T. Piran, Caged black holes: Black holes in compactified space-times. 2. $5 D$ numerical implementation, Phys. Rev. D 69 (2004) 064032 [hep-th/0310096] [InSPIRE].

[48] D.N. Kabat, G. Lifschytz and D.A. Lowe, Black hole thermodynamics from calculations in strongly coupled gauge theory, Phys. Rev. Lett. 86 (2001) 1426 [Int. J. Mod. Phys. A 16 (2001) 856] [hep-th/0007051] [INSPIRE].

[49] S. Catterall and T. Wiseman, Towards lattice simulation of the gauge theory duals to black holes and hot strings, JHEP 12 (2007) 104 [arXiv:0706.3518] [INSPIRE].

[50] M. Hanada, Y. Hyakutake, J. Nishimura and S. Takeuchi, Higher derivative corrections to black hole thermodynamics from supersymmetric matrix quantum mechanics, Phys. Rev. Lett. 102 (2009) 191602 [arXiv:0811.3102] [INSPIRE].

[51] M. Hanada, Y. Hyakutake, G. Ishiki and J. Nishimura, Holographic description of quantum black hole on a computer, Science 344 (2014) 882 [arXiv:1311.5607] [INSPIRE].

[52] D. Kadoh and S. Kamata, Gauge/gravity duality and lattice simulations of one dimensional SYM with sixteen supercharges, arXiv:1503.08499 [INSPIRE].

[53] Y. Hyakutake, Torus-like dielectric D2-brane, JHEP 05 (2001) 013 [hep-th/0103146] [INSPIRE].

[54] T. Azuma, T. Morita and S. Takeuchi, Hagedorn Instability in Dimensionally Reduced Large- $N$ Gauge Theories as Gregory-Laflamme and Rayleigh-Plateau Instabilities, Phys. Rev. Lett. 113 (2014) 091603 [arXiv: 1403.7764] [INSPIRE].

[55] S.S. Gubser and I. Mitra, The Evolution of unstable black holes in anti-de Sitter space, JHEP 08 (2001) 018 [hep-th/0011127] [INSPIRE].

[56] P. Figueras, K. Murata and H.S. Reall, Black hole instabilities and local Penrose inequalities, Class. Quant. Grav. 28 (2011) 225030 [arXiv:1107.5785] [INSPIRE].

[57] S. Hollands and R.M. Wald, Stability of Black Holes and Black Branes, Commun. Math. Phys. 321 (2013) 629 [arXiv:1201.0463] [INSPIRE]. 